\title{
Die Erotik der Macht. Visualisierte Herrscher-Potenz in der Renaissance
}

Herrschen heißt Sich-Selbst-Beherrschen - und damit müßte Herrschen eigentlich auch sexuelle Mäßigung, wenn nicht Enthaltsamkeit bedeuten. ${ }^{1}$ So könnte es beim ersten Blick auf die (offiziellen) Bild- und Textzeugnisse der Frühen Neuzeit, die Wesensart, Lebensführung und Streben von Potentaten zwischen Tugend und Laster für ein größeres Publikum repräsentieren, in der Tat scheinen. Bereits Herkules, der Held schlechthin und zugleich das wohl am häufigsten bemühte Rollenmodell der Zeit für ebendiese Potentaten, wandte sich am Scheideweg selbstverständlich der Virtus zu. Wirkungslos blieben die Lockungen des Vitium - eines Lasters, das in den Bildern der Frühen Neuzeit praktisch immer in der spezifischen Unterkategorie und Gestalt der verführerisch-entblößten Voluptas auftrat. ${ }^{2}$ Statt schneller sexueller Befriedigung wird hier die langfristige Erfüllung durch Aufnahme in den Ruhmestempel der Tugend propagiert. Auch die herausragenden antiken Führergestalten, ein Alexander der Große oder die Scipionen, zeichneten sich durch ihre Selbstbeherrschung und Enthaltsamkeit aus: Die Frauen und Töchter der Besiegten blieben bei ihnen unangetastet, obwohl sie auf diese Beute nach allgemeiner Vorstellung ein ,sexuelles Zugriffsrecht' gehabt hätten. Die gleiche Lust-Disziplin gilt für die intellektuellen Leitfiguren der Zeit wie Sokrates. ${ }^{3}$ Umgekehrt zeigen die zahllosen Beispiele von ,Weiberlisten', wie man selbst höchste Macht durch eigene sexuelle Lüsternheit problemlos verspielen kann. ${ }^{4}$

Die Selbst- und Fremddarstellungen von Herrschern der Frühen Neuzeit als Tugendhelden sind Legion und sie wurden von der Forschung eingehend untersucht. Daß mit genauso großer Selbstverständlichkeit die historische Wirklichkeit und vor allem auch die herrscherliche Sexualmoral vielfach deutlich anders aussah, widerspricht dem nicht - im Gegenteil: Die zentrale Aufgabe der (Selbst-)Inszenierung und ,Image-Bildung' war (und ist) es ja gerade, ein gewünschtes Ideal zu

Ich danke den Mitgliedern der Forschergruppe, voran Beate Kellner, Alexander Kagerer, Christian Kaiser, Jörge Bellin und Dominic Bormann, für die gemeinsamen Diskussionen und wichtigen Hinweise.

1 Reeser 2006.

2 Vgl. nur Panofsky 1930; zur Umdeutung des Herkules auf die Kraft des Volkes dann Hunt 1989, etwa 132-134.

3 Civai 2008.

4 Matthews Grieco 1991; vgl. bereits die großformatigen erotischen Fresken (um 1305/15) im Kommunalpalast von San Gimignano, die als Warnung vor Liebesverstrickungen dienen sollten, dazu Campbell 1997. 
entwerfen, nicht die Kontingenzen des Tatsächlichen zu spiegeln. Allerdings ließen sich beide Bereiche - die fiktionale Welt der Repräsentation und die (von diesen Fiktionen natürlich nie ganz geschiedene) Realität - offenbar gerade auch beim Thema des Herrscherkörpers und seiner Sexualität besonders schwer auseinanderhalten. Mehr noch: Wenn der Herrscher als eine Form des idealen Mannes verstanden werden sollte, ließ sich dies von männlicher Potenz kaum trennen. Hing die Vorstellung von Tugend doch ursächlich mit derjenigen von Männlichkeit zusammen - schon der Begriff für ,Tugend' (virtus) schien aus ,Mann' (vir) hervorgegangen. ${ }^{5}$ Zum Mann-Sein gehörte in diesem Denken jedenfalls unabdingbar aktive Sexualität. Umgekehrt stellt die ,Effeminierung' den größten Würde- und Machtverlust dieses Männlichkeitsideals dar.

Bemerkenswerterweise lieferte wiederum bereits Herkules sowohl für exzeptionelle Potenz wie für verschiedene, Abweichungen ' vom idealen Mann-Sein Musterbeispiele - schon am Mythos und seiner Rezeption läßt sich also erahnen, daß die eingangs formulierte Gleichung vom ,Herrschen als Selbst-Beherrschen ' in der Frühen Neuzeit so unbedingt nicht zutraf. Die (als Warnung zu verstehende) Episode des, verweiblichten' Herkules bei Omphale war ein häufiges Bildthema der Frühen Neuzeit. ${ }^{6}$ Im Ferrara unter Ercole II. d'Este malte Dosso Dossi vor 1542 Gemälde, die in einer komplexen Mischung aus Ruhm und Spott über den Fürsten einerseits den alternden Helden, andererseits dessen herkulischen Körper mit möglicherweise androgynen Qualitäten zeigen. ${ }^{7}$ Bei Hendrick Goltzius wird der Heros dann 1589 zu einem (lächerlich aufgeblasenen?) Muskelmann mit winzigem Geschlecht. ${ }^{8}$ Allein die Erzählung von Herkules als ,Potenz-Protz' scheint erst 1793 von Dominique Vivant Denon in seinem CEuvre priapique visualisiert worden zu sein. ${ }^{9}$ Dabei ist der Bericht, wie der gerade herangewachsene Herkules die 50 Töchter des Königs Thespios schwängert, in der extremsten Version des Pausanias und Gregorius Nazianzenus alle in einer Nacht (wie es auch Vivant Denon darstellt, als „13. Arbeit" untertitelt und den König dabei anzüglich in „Testic" umbenennt;

5 Im verbreitetsten Lexikon des 16. Jahrhunderts heißt es dazu (Calepino 1570, 1164f.): „Dicta est aute[m] Virtus à viro, teste Cic. 2 Tusc. quaest. ... Lactantius lib. de Opificio, Vir nominatus, quod in eo maior vis est quam in foemina: \& hinc Virtus nome[n] accepit." Im Vocabolario della Crusca 1612, 941 wird virtù auch als possanza / potentia sowie vigore, forza und qualità naturara definiert.

6 Bischoff 1998.

7 Halleux 2012; zum größeren Kontext Bulst 2013.

8 Die Deutung des Blattes ist umstritten, vielfach wird es ganz im Gegenteil als politisches Sinnbild der autonomen niederländischen Provinzen interpretiert, aber auch mit anatomischen Lehrbildern und der Rhetorik der Vanitas in Verbindung gebracht, vgl. Krystof 1997, 106-116; zuletzt Melzer 2014, 52f. (Kat. 12). Im Sinne einer pluralen Lesemöglichkeit sei darauf hingewiesen, daß praktisch gleichzeitig Brantôme 1960,172 (Discours sur les femmes mariées, les vefves et les filles a sçavoir desquelles les unes sont plus chaudes a l'amour que les autres) von einer Dame berichtet, die sich über den Kontrast zwischen dem Abguß nach dem riesigen Hercules Farnese in Fontainebleau und der Größe von dessen Geschlecht lustig macht (diese Stelle bereits bei Freedberg 1989, 327).

9 Sandt 1999; Spieth 2001, Bd. 1, 353. 
Abb. 21), unmittelbar mit der ersten großen Heldentat verbunden. ${ }^{10}$ Denn als Belohnung für die (oder nach anderen Versionen zumindest im Zusammenhang mit der) Tötung des Nemeischen Löwen, der das Territorium des Königs Thespios terrorisiert hatte, darf Herkules seine übermenschliche Manneskraft beweisen. Ganz anders als in der Erzählung vom tugendhaft-selbstherrschten Herkules am Scheidewege signalisiert hier gerade die Exuberanz den Halbgott.

Es sind die frühneuzeitlichen Darstellungsformen und Kontexte, in denen die sexuelle Potenz des Herrschers als Signal und Sinnbild für Herrscherpotenz benutzt wurde, die in diesem Beitrag interessieren. Einige Bildbeispiele, die Herrscher und Herrschaft ,sexualisieren', sind von der Forschung schon eingehender diskutiert worden. Es fehlt bislang aber die übergreifende Perspektive auf eine inszenierte

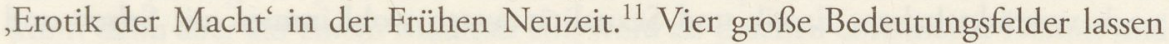
sich dabei - so die Leitthese - unterscheiden: (1.) Sexualität und Potenz des Herrschers konnte - ähnlich wie bei Herkules - eine ,Sonderstellung' durch Transgression der ansonsten geltenden physischen, moralisch-gesellschaftlichen und rechtlichen Grenzen demonstrieren. (2.) In allen metaphorischen Konstruktionen, die Herrschaft in Analogie zu Ehe und Familie und als vermeintlich naturgegeben verstehen wollten, konnte der Potentat in unterschiedlicher Weise in der Position des (sexuell) aktiven Ehemanns und Familienoberhaupts erscheinen, den Untertanen, Städten und Besitztümern war mehr oder weniger explizit die weibliche, passive Rolle zugewiesen. ${ }^{12}$ Zeigen wird sich dabei nicht nur, daß dieser Aspekt häufig wenig trennscharf von dem vorausgehenden zu unterscheiden ist. Wenn man mit Michel Foucault Sexualität insgesamt als Aushandeln und Affirmieren von Machstrukturen begreift, wäre die ,symbolische Sexualität' des Herrschers in vieler Hinsicht gerade nicht als Sonderfall, sondern vor dem Horizont allgemeiner Praktiken zu begreifen. ${ }^{13}$ (3.) Wechselt man von der potenten Wirkkraft zur Wirkung, dann konnte erotische Schönheit und Anziehung des Herrschers für dessen ,Glanz' insgesamt und für die Zuneigung der Untertanen einstehen. Der fließende Übergang von der körperlichen Attraktivität des Herrschers zur (abstrakten) Anziehung auf die Untertanen wurde dabei durch die frühneuzeitliche Vorstellung und Begrifflichkeit der ,Liebe' (amor) ermöglicht: Dieser Terminus umfaßte in fließendem Übergang das gesamte Spektrum vom Geistigen zum rein Körperlichen. ${ }^{14}$ So konnte die allgemein-menschliche Erfahrung von Liebe und körperlichem Verlangen zur allgemeinverständlichen und übertragbaren Chiffre für ein intensives Gefühl werden. (4.) Alle diese Inszenierungsformen, nicht weniger aber auch körper-

10 Gregorius Nazianzenus: Contra Iulianem Imperatorem I; Pausanias: Descriptio Graeciae, 9.27.6-7; vgl. die leicht abweichenden Versionen bei Diodorus Siculus: Bibliotheka, 4.29.2-3; Apollodoros: Bibliotheka, 2.4.9-10; Athenaios: Deipnosophistai, 14.4. 556e-f.

11 Vgl. Ansätze etwa bei Findlen 1994; Bertelli 1995; Zorach 2005; Crawford 2010, v. a. 195-240.

12 Besonders schlagend wird diese Gendermetaphorik in Filarete 1890, 66f. (Buch II, fol. 7v-8r) thematisiert, wo sich der Baumeister in der Rolle der empfangenden Frau versteht, der das , Ideenkind' des Fürsten austrägt und großzieht.

13 Foucault 1976-1984.

14 Vgl. etwa Nagle 1998; Steigerwald 2014. 
liche und sexuelle Unzulänglichkeiten (etwa Häßlichkeit oder Impotenz) sowie sexuelle Devianzen (insbesondere Homoerotik) boten sich schließlich immer auch an, als Mittel der Verleugnung und Schmähung eingesetzt und damit ins Gegenteil verkehrt zu werden.

So wichtig diese ,Erotik der Macht' in der Frühen Neuzeit war - und im übrigen bis heute ist -, die teils gewollten, teils schwierig zu kontrollierenden Ambivalenzen und die besonders im christlichen Kontext moralisch prekäre Thematik scheinen Inszenierung, Thematisierung und Theoretisierung des erotisierten, sexualisierten Herrscherkörpers in mancher Hinsicht gleich auch wieder eingeschränkt oder zumindest auf einer selten verbalisierten, untergründigen Ebene gehalten zu haben. Diese Effekte dürfte das intensivierte visuelle Potential der neuen Renaissancekunst noch verstärkt haben. Erotik und Sexualität waren wohl immer mit Sehen und wirkmächtigen Bildern verbunden gewesen. Vermuten läßt sich gleichwohl, daß die neuen, naturnachahmenden Darstellungsformen des 15./16. Jahrhunderts auch eine neue Stufe in der visuellen Überzeugungskraft erlangten. Mit dieser Überzeugungskraft einher gingen offenbar aber auch alle ,Ambivalenzen der Wirklichkeit: So konnten sowohl ein ideal-paganer Akt wie Giambolognas Neptunstatue vom zentralen Brunnen in Bologna als auch ein ideal-christlicher Akt wie ein Hl. Sebastian des Fra Bartolomeo in sexuellen Traumphantasien der Zeitgenoss(inn)en eine Rolle spielen. ${ }^{15}$ Im Rückblick jedenfalls dürfte nicht zuletzt aufgrund dieser Verkomplizierungen der Eindruck entstanden sein, der Herrscher als Tugendheld, der sich selbst sexuell mäßigt, sei die allein dominierende Vorstellung und das Bild-gebende Ideal der Zeit gewesen.

Kaum betont werden muß schließlich, daß für die hier verfolgte Argumentation komplementär stets auch die ,Erotik der Herrscherin' (Elisabeth I. von England wäre nochmals ein Spezialfall), möglicherweise auch diejenige der Prinzen und Prinzessinnen, der Heerführer usw. in den Blick zu nehmen wäre. ${ }^{16}$ Und überlegen ließe sich zudem, inwiefern andere sexualisierte Rollenmodelle der Zeit - etwa bei Künstlern mit ihren sexualisierten Kreativitätsmetaphoriken oder die ,sexuality of Christ " ${ }^{\text {17 }}$ - nicht zumindest teilweise auch an dem Verständnishorizont einer Erotik der Macht partizipierten. Dies kann hier freilich nicht geleistet werden. Es soll zunächst einmal nur darum gehen, einige Kontexte, Medien und Visualisierungsformen aufzuzeigen, in denen eine Erotik der Macht in der Frühen Neuzeit inszeniert werden konnte.

$15 \mathrm{Zu}$ Giambolognas Neptun vgl. Lavin 1993, 83; Tuttle 2015; zum Hl. Sebastian Fra Bartolomeos S. Freedberg 1989, 346f. - Umgekehrt schreibt Montaigne 1998, Bd. 3, 122 (III/5, Über einige Verse des Vergil'): ,[Livia] pflegte zu sagen, für eine tugendhafte Frau sei ein nackter Mann nicht begehrenswerter als eine kalte Bildsäule“.

16 Vgl. etwa Zanger 1997; Schulte 2002; Paravicini Bagliani 2014. Zum Sonderfall des Papstes vgl. Paravicini Bagliani 1997.

17 Pfisterer 2014; Steinberg 1996. 


\section{Potenz/Transgression}

Der 24-jährige König von Frankreich, Karl VIII., betrieb seine Eroberungspolitik mit vollem Körpereinsatz - und übertraf damit in der Sicht der Zeitgenossen sogar noch Herkules. ${ }^{18} 1494$ marschierte er unter anderem auf Bitten des Herzogs von Mailand in Italien ein, nahm quasi im Vorbeigehen die Akklamation von Florenz entgegen, wurde von Papst Alexander VI. in Rom empfangen, um dann Neapel, die Hauptstadt der Aragonesenherrscher Süditaliens, zu erobern - das eigentlich Ziel des Feldzugs. Angeblich hatte die Krone Frankreichs darauf Rechtsanspruch; zugleich war mit dem Regnum beider Sizilien der prestigeträchtige Anspruch auf den Titel ,König von Jerusalem 'verbunden. In Neapel ritt Karl bei der ersten Ankunft dann aber auf einem Maulesel ein, wohl als Zeichen christlicher Demut und Beschwichtigung der Stadt in Anspielung auf den Einzug Jesu in Jerusalem. Er wollte offenbar von einer Bevölkerung, die den französischen Einmarsch zunächst durchaus mit Hoffnung gesehen hatten, als eine Art ,Erlöser ' und rex pacificus verstanden werden. ${ }^{19}$ Das Blatt wendete sich für Karl allerdings schnell - unter anderem berichten die zeitgenössischen Quellen davon, daß die marodierenden Soldaten so viele Frauen und Mädchen vergewaltigten, daß die Stimmung in der Bevölkerung in Haß umschlug. Der Papst und die Mailänder Sforza, anfänglich Unterstützer Karls, verbündeten sich mit Venedig, Spanien-Aragon und Maximilian I. zur Heiligen Liga und stellten sich den französischen Truppen am 6. Juli 1495 in der Schlacht von Fornovo, deren Sieg von beiden Seiten reklamiert wurde. Die Franzosen zogen sich jedenfalls im Anschluß unter Verlust ihrer gesamten, riesigen Kriegsbeute über die Alpen zurück. ${ }^{20}$

Im zurückgelassenen Zelt Karls fand sich neben vielen Kostbarkeiten auch ein Buch, das gleich von zwei zeitgenössischen Chronisten erwähnt wird: Darin waren schöne Frauen, möglicherweise Prostituierte, verschiedenen Alters und verschiedener Aufmachung porträtiert, mit denen der König in den verschiedenen Städten seines Eroberungszuges Umgang gehabt (oder: die er vergewaltigt) hatte. ${ }^{21} \mathrm{Da}$ die Sammlung keine Erfindung italienischer ,Propaganda' war, zeigt ein Brief bereits vom 11. Juli 1495 an Francesco Gonzaga, in dem unter der Beute auch „li

18 So Marsilio Ficino bei seiner Lobesrede zur Ankunft von Karl VIII. in Florenz: „Superavit \& celebratissimum illum apud omnes Herculem"; zit. nach Schneller 1981-82, 6f.

19 Schneller 1981-82, 46-52; Denis 1979; Antonovics 1995.

20 Santosuosso 1994; zum Verlust eines Reliquiars, das Karl VIII. offenbar als eine Art Talisman mit sich führte, vgl. Jestaz 1989.

21 Corio 1503, fol. Viiì ${ }^{\vee}$, „vi fu trovato un libro nel quale sotto diversi habiti \& etate: al naturale erano depicte molti femine per loro violate in molte citate: e seco il portavano per memoria“; vgl. den im Wortlaut sehr ähnlichen und jedenfalls vor 1512, dem Todesjahr des Autors, niedergeschriebenen Augenzeugenbericht des Benedetti 1549, fol. $31^{\mathrm{r}}$ [eigentlich 23]: „In quella preda vidi io un libro, nel quale erano dipinte varie imagini di meretrici sotto diverso habito, \& età, ritratte al naturale; secondo che la lascivia, \& l'amore l'haveva tratto in ciascuna città: queste portava egli seco dipinte per ricordarsene poi." Beide Quellen zitiert bei Campbell 1990, 209, Anm. 88; zur späteren Tradition von Kurtisanen-Porträts vgl. Filzmoser 2014. 
retracti di quelle damiselle del re" erwähnt werden. ${ }^{22}$ Die Bildnisse sind freilich verloren und es ist auch kein Vergleichsbeispiel aus der Zeit um 1500 bekannt, das eine genauere Vorstellung davon vermitteln könnte. Diente dieses Buch laut Chronisten der Erinnerung des Königs an sein außerehelichen Liebesabenteuer, so möchte man ein anderes, ebenfalls in Feindeshand gefallene Bildnis - das seines erstgeborenen, mit 26 Monaten porträtierten Sohnes, das ihm ins italienische Heerlager nachgesandt worden war - zunächst als Ausdruck von väterlichem Stolz und Liebe verstehen. ${ }^{23} \mathrm{Daß}$ das Kinderbildnis zugleich und mindestens so sehr auch die herrscherliche Potenz und den Fortbestand der älteren Linie der Valois bezeugen sollte (bevor der Kleine 1495 überraschend an Masern starb), läßt der Umstand erahnen, daß es in einem silbernen Behältnis zusammen mit dem Siegel von Karl und dem Siegel von dessen Vater, Ludwig XI., aufbewahrt wurde. ${ }^{24}$

$\mathrm{Zu}$ fragen ist, ob nicht auch das Buch mit den Frauenbildnissen noch anderes als private Lustbefriedigung bezweckte? Sollten nicht die vielfältigen Erfolge seiner Manneskraft auch die vielfältigen Eroberungserfolge seines Feldzugs spiegeln oder anders formuliert: sollte es sich nicht auch um ein repräsentatives Bildzeugnis für einen ausgewählten Männerkreis dafür handeln, daß die Potenz des französischen Königs Italien auf allen Ebenen eroberte und unterwarf? Das Erobern wie der Einzug des Herrschers in seine Stadt ließen sich Europa vielfach als eine Art von (Braut-)Werbung, sexueller Unterwerfung und Geschlechtsverkehr verstehen - der Besitz gibt sich gewollt oder gezwungen dem neuen Herrn hin. Auch im umgekehrten Sinne (Eroberung als Metapher für den Liebeskampf) finden sich zahlreiche Indizien dafür - sowohl in Bildform: der Sturm auf die Minneburg, wie in Redewendungen, die den Geschlechtsverkehr mit Eroberungs- und Einzugsmetaphern umschreiben: „mettere il Soldano in Babilonia“, „ponere lo Papa a Roma“ oder dann im Deutschen: „nun wollen wir Konstantinopel stürmen“. 25

Mehr noch: Das gewaltsame Gefügig-Machen einer Frau ließ sich möglicherweise ganz allgemein auf die aggressiv-erfolgreiche Aktivität des jungen Königs beziehen, der sein Glück in die richtigen Bahnen zwang. Etwas später heißt es in Niccolò Machiavellis Il Principe (1513) in diesem Sinne: „Ich halte es für richtig, lieber zupackend als ängstlich zu sein. Denn das Glück ist eine Frau, und um sie unten zu halten, muß man sie schlagen und niederwerfen. Und man sieht ja, daß sie sich häufiger von solchen besiegen läßt als von jenen, die kontrolliert vorgehen. Und wie jede Frau ist sie [das Glück] die Freundin der Jungen, weil diese weniger rücksichtsvoll sind und wilder und ihr mit mehr Wagemut befehlen." ${ }^{26}$ Und zu-

22 Brief von Benedetto Capilupi, publiziert von Luzio/Renier 1890, 631.

23 Heute Jean Hey zugeschrieben, vgl. Campbell 1990, 214 und Anm. 105.

24 Malipiero 1843, 371: „Andrea Zantani, Podestà di Ravenna, ... ha trovà una cassetta massizza d'arzento, tre quarte longa; e do larga, fodrà de veluo celeste. Ghe era dentro un sigillo d'oro massizzo del Re de Franza modern, e un altro mazor, pur d'oro, che fo de so padre; e un retratto de so fio de età de tre anni."

25 Müller 1999; Rank 1914. - Vgl. für antike und mittelalterliche Anwendungen dieser Ideen etwa Sueton: Divus Iulius 7,2 und Dante: Epistola VII,7.

26 Machiavelli 2013, cap. 25; vgl. Fenichel Pitkin 1984. 
mindest Karls zweiter Nachfolger, Franz I., ließ sich wohl 1537 von Benvenuto Cellini eine Medaille fertigen, auf deren Rückseite der Herrscher eine nackte weibliche Fortuna niederreitet und -knüppelt. ${ }^{27}$

Schließlich wäre sogar eine genealogische Assoziation für Karls demonstrativ exzessives Liebesleben denkbar: Die Machtbasis des ersten Kapetingers, Hugo, wird zumindest in der Ausdichtung der Chansons des gestes und der deutschen Übersetzung Hug Schapler auch in der Fülle seiner unehelichen Kinder aus der Zeit vor Herrschaftsantritt gesehen. ${ }^{28}$ In einer Zeit, da die auf die Kapetinger nachfolgende Nebenlinie der Valois nicht gerade mit vielen Söhnen gesegnet war, könnten also das Bildnis des kleinen Thronfolgers und der Prostituierten gemeinsam für die Potenz des Königs eingestanden haben.

Vielleicht das stärkste Indiz dafür, daß Karls ,Schönheitsgalerie‘ von 1494/95 tatsächlich auch im Sinne einer sexualisierten Herrschaftsrepräsentation verstanden werden sollte, liefern jedoch die in mancher Hinsicht vergleichbaren Sammlungen seiner beiden Nachfolger. Überliefert ist, daß der Mailänder Herzog Galeazzo Maria Sforza bereits 1473 eine Porträtsammlung schöner (junger und offenbar heiratsfähiger) Mädchen seines Herrschaftsgebietes anlegen ließ. ${ }^{29}$ Neben praktischen und die Augen, erfreuenden' Zwecken könnte man dies versuchsweise auch mit der Tradition in Verbindung bringen, Italien als Ganzes, aber auch in seinen einzelnen Gegenden weiblich zu personifizieren. Die Mädchen würden so für den Herrschaftsbereich, einzelne Städte und Dörfer einstehen. Als dann Ludwig XII., der Nachfolger Karls VIII., im zweiten französischen Italienfeldzug 1499 Mailand erobert, wird eine Porträtgalerie von Mitgliedern und Verbündeten des Hauses Sforza (nun auch mit Bildnissen junger Männer) nach Ambroise überführt. Auch hier scheint neben anderen Gründen ,symbolische Aneignung und Unterwerfung nicht unwichtig. ${ }^{30}$ Noch offensichtlicher wird dies an einer Sammlung von $27 \mathrm{zu}$ einem Buch zusammengebundenen, namentlich bezeichneten Bildnisminiaturen Mailänder Frauen, die Franz I. wohl um 1518 erhielt - von wem genau ist unbekannt (Abb. 22). Das Vorwort des kaum bekannten Malers Giovanni Ambrogio Noceto bezeichnet die Dargestellten als „schöne Schar von Nymphen“, die offenbar den König, dessen Porträt die Folge eröffnet, umringen. ${ }^{31}$ Im Unterschied zu diesem sind dann alle nachfolgenden Frauenbildnisse durch einen Klappdeckel verborgen. Die ersten sieben, die früh verwitwete Frauen zeigen, sind mit den Namen der sieben Tugenden versehen. Entscheidend scheint die doppelte Zielrichtung dieser Zusammenstellung, die mit ihrer idealen Tugendordnung einerseits den Herrscher auf seine Stadt verpflichtet, andererseits die erotische Illusion erzeugt, dieser könne exklusiv über die schönsten Frauen verfügen. Oder anders formuliert: Auf die Unterwerfungsgeste der Stadt, ihre schönsten und tugendhaftesten

27 Attwood 2004

28 Haug 1989.

29 Dazu Wenzel 2001, $95 f$.

30 Dazu und dann auch zum folgenden Beispiel Wenzel 2001, 100-113.

31 Mailand, Codex Trivulziano N. 2159; die kommentierte Faksimilieausgabe Bologna 1989. 
Frauen als eine Art visuelle Beute und ,Geiseln im Bildnis` dem fremden, potenten Herrscher zu präsentieren, sollte dieser wie ein neuer Alexander oder Scipio mit tugendhafter Selbstbeherrschung reagieren.

Daß Franz I. für diese Art von (diplomatischen) Geschenken sehr empfänglich war (oder zumindest die italienischen Geber dies dachten), zeigen noch andere Beispiele: Kardinal Bernardo Dovizi da Bibbiena, Gesandter des Papstes am französischen Hof, überreichte das Bildnis der Vizekönigin von Neapel. Auch hier dürfte es nicht nur darum gegangen sein, sich am Konterfei einer herausragend schönen Frau, gemalt vom besten Maler Italiens, Raffael (unter Mithilfe von Giulio Romano), zu erfreuen. Die Vizekönigin war zudem die Gattin des einen Mannes, gegen den Franz auf seinem Feldzug in Italien eine Niederlage hatte hinnehmen müssen. Möglicherweise kam daher zur Lust an weiblicher Schönheit auch das Gefühl dazu, über den Konkurrenten zumindest durch den Blick auf dessen Frau triumphieren zu können. Daß dies nicht nur modernes Psychologisieren ist, läßt sich an anderen, dokumentierten Reaktionen zumindest ansatzweise belegen: So überreichte der Mantuaner Gesandte Franz zur gleichen Zeit ein Aktbildnis sowie ein Begleitschreiben des Markgrafen von Mantua, in dem dieser dem französischen König als „großem Experten in Fragen körperlicher, vor allem weiblicher Schönheit" schmeichelte. Der König konnte sich - so berichtete der Gesandte seinem Herrn - an diesem Geschenk kaum satt sehen und habe sich sofort erkundigt, ob eine Hofdame der Markgräfin als Aktmodell gedient habe. ${ }^{32}$

Vor diesem Hintergrund gewinnt auch der Vorschlag an Überzeugungskraft, eine Darstellung der späteren Niederlage von Franz I. bei Pavia 1525 gegen Karl V. als visuelle Anspielung auf sexuelle Demütigung und Verweiblichung zu verstehen: Ein Maiolica-Teller des Francesco Xanto Avelli, der rückseitig mit „1534. Caddette il Re Cristiâ sotto Pauia... .F.X" beschriftet ist, könnte mit der Haltung des rückwärts vom Pferd gefallenen französischen Königs auf die erste Stellung in Giulio Romanos und Marc'Antonio Raimondis I Modi anspielen. ${ }^{33}$ Franz wäre damit als verweiblicht-passiv gegenüber dem siegreichen Karl diffamiert. Solche Polemiken der ,Effeminierung ' hatten im übrigen die Propaganda auf italienischer wie französischer Seite seit dem Einfall Karls VIII. geprägt. So riefen die Italiener ihre Landsleute nicht nur auf, die weiblichen, verweichlichten Sitten abzulegen und wieder zu römisch-männlicher Härte zurückzukehren; zumal gegen einen so häßlichen und verwachsenen Angreifer wie Karl VIII., „più simile a mostro che a uomo", dem die zeitgenössischen Physiognomiker zugleich eine besonders, lüsterne Natur' nachsagten. ${ }^{34}$ Wer immer sich mit den Angreifern einließ, wurde jedenfalls mit dẹm neuen Übel des mal francese, der Syphilis, bestraft.

Umgekehrt könnte dann die Konzentration von Franz I. auf die im Anschluß an das italienische Desaster konzipierte und in Angriff genommene Schloßanlage von

32 Dazu Fritz 2002.

33 Findlen 1994, 98-101.

$34 \mathrm{Zu}$,Verweichlichung und ,Härte' vgl. Springer 2010, v. a. 15-20; zur erstmals 1504 gedruckten Deutung des Bartolomeo Cocles (cap. II/4) vgl. Ziegler 2004. 
Fontainebleau mit ihrer teils hocherotischen Ausstattung auch als eine Art von visueller, ,überkompensierender ' Demonstration der so in Mitleidenschaft gezogenen herrscherlichen Potenz verstanden werden. ${ }^{35}$

Die Idee, als ,erfolgreicher Mann' zu Beginn des 16. Jahrhunderts die eigene Macht in Form von offensiven sexuellen Potenzdemonstrationen auszustellen, war auch schon vor Franz I. südlich wie nördlich der Alpen bekannt. Federico II. Gonzaga, Markgraf von Mantua, ließ ab 1524 vor den Toren Mantuas den Palazzo del Te als villa suburbana und Ort des Vergnügens errichten - konkret biographisch als, Liebesnest' für seine Mätresse. Allein darin wird man kaum den letztendlichen Grund für die umfangreiche Bildausstattung des Palastes sehen wollen; vielmehr nutzte der Markgraf den Ort, um spielerisch-allusiv für seine Besucher ein alternatives Idealbild des männlichen Herrschers zu entwerfen, der verschiedene antike Rollenmodelle übernehmen konnte: so etwa auch die des Dauer-Liebhabers Jupiter, der sich in kaum zu überbietender visueller Eindeutigkeit der sterblichen Olympias näherte und mit ihr Alexander den Großen zeugte (Abb. 23). ${ }^{36}$

Im Norden hatte Philip von Burgund, Admiral der Niederlande und dann Bischof von Utrecht, nach Ausweis des erhaltenen Inventars seines Palastes gezielt erotische mythologische Bilder gesammelt. Besonders offensichtlich wird bei einem fast lebensgroßen Gemälde von Neptun und Amphitrite des Jan Gossaert (um 1516/18) aufgrund der Imprese und des abgekürzten Namenszuges Philips im Bild die Identifizierung des antiken Meeresgottes mit dem Admiral, wie sie auch zeitgenössische Panegyriken aufrufen. ${ }^{37}$ Verweisen ließe sich aber auch auf die Sammlung mythologischer Erotica der Herzöge von Sachsen im Schloß von Wittenberg, auf die erotischen Correggio-Gemälde der Gonzaga oder dann später auf die ,Nuditäten'-Kammern Philips II. in Madrid oder Rudolfs II. in Prag: Daß etwa Philip in Tizians Venus mit Orgelspieler von 1548/49 sogar selbst in der Rolle des Musikers auftritt, belegt die zunehmende Wirkmacht der Vorstellung, daß der ideale Herrscher zugleich ein vollkommener Liebhaber und Kenner von körperlicher Schönheit sein müsse. ${ }^{38}$

Wenn wie bei den französischen Königen in Fontainebleau die Bildersammlung gleich auch noch im Badetrakt hing, verstärkten sich Inszenierung im Bild und Inszenierung des Körpers noch gegenseitig. ${ }^{39}$ Schlagendes Beispiel für die Bedeutung des nackten Herrscher-Körpers im Bad war bereits der böhmische König Wenzel IV. (bis zu seiner Absetzung 1400 auch römisch-deutscher König) gewesen, der um 1400 nicht nur ein Pracht-Exemplar der Goldenen Bulle, sondern auch eine für ihn gefertigte Bibel mit Randszenen versehen ließ, in denen er nackt von

35 Wilson-Chevalier 1993; nicht immer ganz überzeugend Waddington 2001; Tauber 2009; Crawford 2010, 204-207.

36 Verheyen 1977; Talvacchia 1999, 45-47.

37 Schrader 2010.

38 Fabiański 1998; Prohaska/Ferino-Pagden/Wied 1995; Borggrefe 2006.

39 Eschenfelder 1991. 
Bademädchen bedient wurde. ${ }^{40}$ Die Forschung verweist neuerdings zur Erklärung dieser irritierenden Darstellungen unter anderem auf einen vom König begründeten Badeorden, bei dem es um spirituelle Reinigung ging. Gleichwohl ist die erotische Qualität dieser prominenten Darstellungen kaum zu leugnen und scheint entscheidender Ausdruck für Wenzels (möglicherweise schon für die Zeitgenossen schwer akzeptables) ,körperbetontes' Selbstverständnis. Im Rom des frühen 16. Jahrhunderts versuchten sich dann Papst und Kardinäle in der Erotik ihrer Badezimmerausstattungen zu übertreffen. ${ }^{41}$ An der Wende zur Neuzeit dürfte so möglicherweise der ,badende Souverän' mit seiner erotischen Aufladung noch wichtiger gewesen sein als die Machtdemonstrationen des „schwimmenden Souveräns“ “. 42

Erst die hier skizzierten Kontexte von Potenz und Transgression als ,Herrschaftszeichen' erlauben schließlich auch den Brauch zu verstehen, einem Potentaten beim Einzug oder Empfang Frauen nackt zu präsentieren. So trugen etwa beim Einzug Ludwigs XI. in Paris 1461 drei junge Frauen vollständig nackt Festgedichte vor. ${ }^{43}$ Karl der Kühne durfte sich 1468 in Lille eine mythologische Aufführung zum „Urteil des Paris" mit drei nackten Göttinnen ansehen - und das gleiche mythische Thema wurde 1494 bei der Krönung Philipps des Schönen in Antwerpen und nochmals 1496 beim Einzug seiner Frau Johanna in Brüssel zum Besten gegeben (Abb. 24). ${ }^{44} \mathrm{Ob}$ man beim letzten Beispiel ein spezifisch weibliches Programm ausmachen kann, sei hier dahingestellt - exzeptionell war auf jeden Fall die anschließende Szene mit einem „Haus der Freude und des Vergnügens", in dem sich alle aus Begeisterung über die anstehende Fürsten-Hochzeit sinnlichen Vergnügungen hingaben. ${ }^{45}$ Nacktheit war auch noch in anderen Kontexten bei weiblichem Publikum zu finden, so bei einem Bankett in Venedig für die Königin von Ungarn, bei dem unter anderem eine nackte Sirene zu sehen war, die sich selbst malte (fraglich bleibt, ob es sich um eine lebende Person handelte oder um eine Zuckerskulptur). ${ }^{46}$ Die Metaphorik der Stadt (und des ganzen Landes) als erwartungsvollfruchtbare Jungfrau, die sich ihrem Bräutigam und ,neuen Herkules' hingibt, wurde etwa beim Einzug der zukünftigen Königin Maria de’ Medici in Avignon herausgestellt (Heinrich IV. kam nicht rechtzeitig an, ihm wurde aber von Jupiter in dieser Festdekoration als Auftrag mitgegeben: „Semez au Jardin de Florence"). In der Festbeschreibung des André Valladier wird die sechste Station, der Tempel des Janus, als Höhepunkt des Ganzen beschrieben. Hermen neben dem Haupteingang

40 Theisen 1999.

41 Contardi/Lilius 1984.

42 Vgl. Bredekamp 2014.

43 Couderc 1896.

44 Cauchies 1998; Legaré 2011; Vandenbroek 2012.

45 „domus deliciae et iocunditatis // Hoc scemate representatur qui uti hii deliciosi cunctis se voluptatibus occupaverunt. Sic occasione coniunctionis Philippi et Johannae ducum omnibus tristiciis sese singuli exuentes cunctis iocis indulserunt." Zit nach Vandenbroeck 2012, 179-181.

46 Dazu Brown 2011, 34. - Für eine seltene Bildüberlieferung eines solchen Tafelspiels aus dem Burgund der 1480/90er Jahre (eine Zeichnung im Kupferstichkabinett Berlin, KdZ 1983) mit einer wohl lebenden nackten Frau vgl. Marti 2008, 294 (Kat. 117). 
resümierten die bisherigen Triumph-Bögen und ihre Themen. Zur dritten Herme, die für den „fruchtbaren Garten Frankreichs“ stand, heißt es: „Le tiers faisoit une fille feconde $\&$ fertile chargee de fruictage, $\&$ des festons pendants soubs ses poupes pendantes, \& fecondes “. 47 Einen vorausgehenden Höhepunkt dieser Potenz-Demonstrationen hatte bereits der Krönungseinzug Heinrichs IV. in Paris 1594 geboten, wo - nachdem schon Heinrich II. bei seinen Einzügen Anspielungen auf die Geliebte Diane de Poitiers in der ephemeren Festdekoration geschätzt hatte - nun gleich die Maîtresse Gabrielle d'Estrées mit ihrem eben geborenen Sohn auf einem Wagen mitgeführt worden war. ${ }^{48}$

Das berühmteste Beispiel diese sexualisierten Herrscherempfänge dürfte aber der Einzug Karls V. in Antwerpen 1520 gewesen sein, den Dürer als Augenzeuge miterlebte. Bereits 1515 hatten die italienischstämmigen Einwohner beim Entrée Karls in Brügge versucht, durch die "ungewohnte Neuheit“ eines Brunnens mit drei Statuen nackter Frauen, aus deren Brüsten Weißwein, Rotwein bzw. Rosenwasser strömte und deren Ausführung "trespres du vif et dartifice tant parfaict“ war, die wohlwollende Aufmerksamkeit des Herrschers auf sich zu lenken. ${ }^{49}$ Fünf Jahre später in Antwerpen notierte der Nürnberger Maler dann selbst in seinem Reisetagebuch zwar nur kurz etwas von „schöne jungfrauen bilder“. Wieder daheim, scheint er jedoch Philipp Melanchthon ausführlicher berichtet zu haben, denn dieser überliefert unter Verweis auf Dürer, daß unter dem Begleitpersonal des Festzuges eine Gruppe bis auf einen hauchdünnen Schleier nackter junger Frauen zu sehen gewesen war. Der Reformator macht daraus ein Exemplum für kaiserliche modestia und legt Dürer zudem in den Mund, daß der junge Kaiser kaum hingesehen, er als Maler dagegen die schönen weiblichen Gestalten ungeniert und eingehend studiert habe. ${ }^{50}$ Der Empfang des Kaisers - der bei seinem Einzug auf den dreizehn Schau-Bühnen unter anderem auch noch die Drei Grazien und Herkules/ Karl am Scheidewege zwischen Tugend und Laster zu sehen bekam - bringt nicht nur die hier über mehrere Jahrzehnte verfolgte Vorstellung auf den Punkt, daß sich ein Land oder eine Stadt in erotisiert-sexualisierter Gestalt dem allpotenten Herrscher unterwirft und anbietet, um ihre natürliche Erfüllung zu finden. Melanchthons Schilderung der Ereignisse läßt auch vermuten, daß bei dem Ereignis in Antwerpen 1520 unterschiedliche kulturelle Traditionen und Erwartungen aufeinander trafen und die ,Erotik der Macht ${ }^{\star}$ eben doch keine überall gleichermaßen geschätzte Vorstellung war. Der junge, 20-jährige Karl V. verhält sich offenbar vollkommen anders als es wohl der junge, 24-jährige Karl VIII. von Frankreich getan hätte: Der Kaiser betreibt ,Blick-Disziplin` und sieht die nackten Mädchen

47 Valladier 1600, 145 unter Rückbezug auf den Bogen, der ab Seite 117 beschrieben wird; die Tafel 143 zeigt die Hermen in veränderter Reihenfolge. - Für vorausgehende Fruchtbarkeits-Symboliken in allegorischen Texten vgl. etwa Hochner 2006, 197-205; für frühere Einzügen vgl. Crawford 2010, 208-214; später dann Zanger 2002.

48 Vgl. Ramsey 2007; vgl. zu anderen Formen des erotisierten Auftritt eines französischen Königs im Bild etwa Jollet 2006.

49 Dupuys 1515, fol. E5'; dazu Philipp 2011, 160.

50 Dürer 1956, 327; die Tagebuch-Notiz 158. 
kaum an. In der Tradition der süddeutschen und österreichischen HabsburgerLande und dann vor allem auch bei den Protestanten scheint - diesem Beispiel nach zu schließen - ,politische Erotik ${ }^{`}$ zunächst kein positiver Ausweis von Herrschermacht gewesen zu sein.

\section{Liebe/ Verlangen}

Mit Thomas Hobbes' Leviathan (1651) gewinnt auch die Vorstellung von der Erotik der Macht eine neue Differenzierung. Lag in den bislang vorgestellten Beispielen die Aufmerksamkeit fast ausschließlich auf der Aktivität und (tatsächlichen oder vorgeblichen) Potenz des Herrschers, so definiert Hobbes ,Macht' auch explizit über die (doch eher passive) Wirkung auf andere und über Zuschreibungen. So gehört in den einen Bereich der „zweckdienlichen Macht“ eben auch, „im Ruf von Macht zu stehen“. Und in den anderen Bereich der "natürlichen Macht" fällt neben physischer Stärke etwa körperliche (und offenbar erotisierende) Schönheit, „denn da sie Gutes verspricht, erwirbt man dadurch leicht die Gunst von Frauen und Fremden". ${ }^{51}$ Eine solche Liebe und erotisches Verlangen erweckende und damit zugleich Verfügungs- und Handlungsmacht demonstrierende Schönheit des Herrschers spielte allerdings - das versucht dieses Kapitel plausibel zu machen auch schon vor Hobbes eine Rolle in der visuellen Repräsentation.

Der schöne Körper erwies sich zunächst auf mehreren Ebenen als ambivalent. Einerseits verstand man in den Traditionen antiker kalokagathia-Vorstellungen und der Physiognomik den schönen Körper als Spiegel eines ,schönen', tugendhaften Geistes: „Denn es ist unmöglich, daß in einem verzogenen und mißgebildeten Körper eine schöne und reine Seele wohnen kann, denn da der Körper das Organ der Seele ist, muß notwendigerweise der innere dem äußeren Menschen entsprechen. " ${ }^{2}$ Andererseits lehrten zumindest die Theologen und die historische Erfahrung das Gegenteil. In Diego de Saavedra Fajardos berühmtem emblematischem Fürstenspiegel (zuerst Spanisch 1640), der in vieler Hinsicht die tradierten Gedanken zusammenfaßt, werden nicht nur diese Argumente gegeneinander abgewogen, sondern immer wieder auch explizit auf die Disziplinierung der Sexualität bezogen: „Und gewißlich eine schöne geschickte zusamenfügung der glider / und löbliche farbe / verwundet mit freundlichen pfeilen die augen / welche / wan sie ins gemüht

51 Hobbes 1996, 66f. (Kap. 10).

52 Diese Idee wurde etwa mehrfach und besonders explizit zu Beginn des 16. Jahrhunderts von Bernardino Cacciante formuliert: „Perché impossibile cosa è, che in un corpo distorto et malcompacto, gli possa habitare una anima bella et pura, perché il corpo, essendo organo de l'anima, gli è necessario che lo interiore huomo allo esteriore se assimiglie." Zit. nach Martini 1982, 31; vgl. bereits Guarino da Verona: De vocabulorum observatione (zit. nach Baxandall 1971, 14): „virtus enim est et pulchritudo in vilem ponuntur, sicuti etiam e contra malatia et vitium pro deformitate“. Allerdings läßt sich auch die gegenteilige Vorstellung nachweisen, etwa bei Mirandula 1544, fol. 184" : „Ingenium exornat saepe corporalem deformitatem, ..." unter Verweis auf Ovid und Titus Calphurnius. - Zum Spektrum der Schönheits-Vorstellungen der Renaissance etwa AmesLewis/Rogers 1998, und Cropper 1995. 
tieffer hinein gelassen werden / nehmen sie des Menschen vornehmen und willen gäntzlich ein." Es folgen jedoch gleich auch Gegenargumente und Warnungen: „Die Hoffart und uberhebung wegen die gestalt die thun leichtlich die kräfften der tugenden außreitten und hat sich der Fürst fleissig vorzusehen / daß er sich nicht einer furwitzigen weiber schönheit / welches anderen eine anreitzung zur geilheit ist gebrauche / sondern eine solche so die wahre tugenden pflegt ... " 53 Für die Bilder bei Hof, die nicht Tugend-Exempla vorstellen, gilt schließlich: „Wie thun die gemählder des unreinen Jovis zur unzucht entzünden? und gewiß in derogleichen thut unsere verderbte Natur / sich viel künstlicher erzeigen / als in den züchtigen / unnd zwahr erstlich werden die künst Stücke wegen der vortreffligkeit des Meisters hoch gehalten / hernacher aber / werden die wände nuhr damit gekleidet / auff daß die geilheit die augen erfrisschen ..." 54

Zahllose Bildwerke des 15. und 16. Jahrhunderts scheinen mehr oder weniger bewußt die Herrschaftsrepräsentation mit der Wirkung erotisierender Körper in Verbindung zu bringen. Eine genaue historische Rekonstruktion und Abgrenzung der individuellen Intentionen und Sehverhalten dürfte freilich kaum mehr möglich sein. Zumal die neuen, naturnahen Darstellungsmittel nicht nur eine viel größere, visuell überzeugende körperliche Präsenz des Bildwerks erzeugen konnten und etwa erlaubten, zwischen verschiedenen Altersstufen sehr präzise zu unterscheiden, ${ }^{55}$ sondern auch bestimme Charakteristika, Vorzüge wie Defizite, nun viel deutlicher ausstellten oder Möglichkeiten des Ausstellens eröffneten. Das Verhältnis von Idealisierung und Wirklichkeit für die erotische Attraktivität des Herrscherkörpers im Bild galt es neu auszuhandeln.

Besonders offensichtlich erweist sich dieser neue Aushandlungskontext an Bildwerken, die das männliche Geschlecht darstellten oder andeuteten. So bekrönten etwa das Grabmal des Dogen Andrea Vendramin in SS. Giovanni e Paolo zu Venedig, das Tullio Lombardo in den 1480er Jahren mit seiner Werkstatt errichtete, zwei jugendliche Schildhalter. Nackt bis auf ein eng anliegendes Lendentuch, unter dem sich überdeutlich das Geschlecht abzeichnet und über dem detailliert die Schambehaarung zu erkennen ist, stellt sich die Frage nach dem Sinn dieser spektakulären Figuren, selbst wenn ihre relativ hohe Aufstellung etwas von dem Effekt verschleiert haben dürfte (Abb. 25) $?^{56}$ Denkt man an andere mögliche Wappenhalterfiguren: Krieger, heraldische Tiere, Wilde Männer und ihre Konnotationen, dann läßt sich auch in diesem Fall vermuten, daß hier die jugendliche Schönheit und Potenz der Körper als ,allegorische Auslagerung, Kommentierung und Disziplinierung' der Macht und Tugenden des Dogen verstanden werden sollte. Nicht auf Begleitfiguren ,ausgelagert', sondern idealisierend, überhöht' ist das Geschlecht auf Bronzinos Por-

53 Zit. nach der ersten deutschen Übersetzung; vgl. Saavedra Fajardo 1655, 26f. (Symbolum III)

54 Saavedra Fajardo 1655, 18 (Symbolum II). Die Vorstellung, erotische Bilder würden größere Kunstfertigkeit zeigen und diese vorantreiben, geht auf Plinius: nat. 33, 2,4 zurück.

55 Vgl. etwa Koos 2006.

56 Knuth 2007; Markham Schulz 2014, 46-66; zur ,Ausstellung' des Geschlechts Bertelli 1995, 153-160. 
trät des Andrea Doria (ca. 1550/55). Auch wenn das Gemälde den erfolgreichen Admiral zunächst nicht als Neptun, sondern als antik-römischen Flottenführer mit Ruder zeigte (wann genau dieses in den Dreizack Neptuns umgewandelt wurde, ist unklar): Heroische Nacktheit und das im Ansatz zu sehende Geschlecht stehen hier für Potenz und Sieg. Hinzuweisen wäre zudem auf ein Verständnis von Nacktheit als Tugendsignal, wie es etwa Lomazzo in seinem Malereitraktat von 1584 formulierte: „Manchmal ließen [die antiken Kaiser] sich auch nackt darstellen, um dadurch anzuzeigen, daß der Kaiser frei sein muß und dem Volk das, was er ist, offen zeigen muß und daß er für seine Güte verehrt und für die Gerechtigkeit, die er walten läßt, gefürchtet werden muß. " 57 Das Beispiel der anderen (geplanten wie teils ausgeführten) Monumente für Andrea Doria läßt allerdings vermuten, daß bereits in den 1540er Jahren und parallel zur einsetzenden Gegenreformation die Wahrnehmung männlicher (heroischer) Nacktheit in die Kritik geriet. Eine Statue, die Doria nun tatsächlich in der mythologischen Rolle von Neptun zeigen sollte und zunächst komplett nackt geplant war, wurde mit verhüllendem Tuch ausgeführt.

Eine ähnliche ,Zensur` im Laufe des Ausarbeitungsprozesses läßt sich auch an der Bronzestatue für Karl V. von Leone Leoni vermuten. 1549 hatte der italienische Bildhauer in Brüssel den Auftrag für dieses Bronzemonument des Kaisers erhalten. Bis 1553 entstand ein überlebensgroßes Standbild des heroisch nackten Karl, der über den Furor triumphiert (Abb. 26). Im August 1555 ist dann erstmals von einem abnehmbaren Panzer der Statue die Rede, der es erlaubte, den Körper des Kaisers weitestgehend unter der Rüstung zu verbergen. Giorgio Vasari rühmt in seinen Künstler-Viten 1568 diesen Einfall zwar als besonders erfindungsreich. ${ }^{58}$ Gleichwohl bleibt der Eindruck einer nachträglichen Korrektur der allzu offensiven Nacktheit, zumal sich der Kaiser bis zu seinem Tod 1558 offenbar nicht sonderlich um das spektakuläre Bronzestandbild kümmerte. 1556 wurde es zwar nach Madrid überführt, aber erst 1564 erfolgte die Ziselierung - es blieb freilich weiterhin in der Werkstatt von Leone Leonis Sohn Pompeo bis zu dessen Tod 1608. Erst danach wurde die Gruppe im Garten des Buen Retiro-Palastes aufgestellt. Möglicherweise also konnte Karl V., der sich zunehmend ins Kloster des Escorial zurückzog, bis zum Ende seines Lebens mit dem Konzept eines erotisierten Herrscherkörpers, wie es der italienische Bildhauer entwarf, nichts anfangen.

In der Stilisierung nicht der Bildwelten, sondern der Wirklichkeit scheint im übrigen die Verwendung der Schamkapsel als Bestandteil der Männermode des 15. und 16. Jahrhunderts eine entsprechende Geschichte zu durchlaufen. Schon ihr Aufkommen wurde von ablehnenden Kommentaren begleitet; es häufen sich dann aber die negativen Urteile über die Hosenmode ab der Mitte des 16. Jahrhunderts. So wird etwa 1555 in Andreas Musculus' Hosen-Teuffel oder dann im dritten Band von Montaignes Essais (1586/87) kritisiert, daß das Geschlecht nicht verborgen,

57 Zit. nach Baader 1999; zum Kontext Himmelmann 1985.

58 Vasari 1987, 201. - Vgl. Poeschke 1992, 226f.; Coppel Aréizaga 1998, 66-72 (Kat. 10). 
sondern zum Laster reizend ausgestellt, ja „durch betrügerische Manipulation über sein natürliches Maß" hinaus noch vergrößert würde. ${ }^{59}$

Ungeachtet dieser zunehmenden Ablehnung des nackten (Herrscher-)Körpers werden gleichwohl 1565/73 die Statuen von Heinrich II. und Katherina de' Medici auf ihrem Doppel-Grabmal in St. Denis als nacktes, nebeneinander schlafendes Liebespaar mit erotischen Idealkörpern dargestellt - ein größerer Gegensatz zu den bis dato gebräuchlichen, von Würmern zerfressenen Transis läßt sich kaum vorstellen. ${ }^{60}$ Es hat einiges für sich, daß hier die Auferstehungsleiber des Herrscherpaares vorweggenommen werden, deren zukünftige Idealität eben auch in ihrer erotischen Attraktion zur Anschauung gebracht werden sollte.

Wie sich die zirkulären Anziehungskräfte der Liebe auch jenseits sexualisierter Körper-Darstellungen als Herrschaftsargument einsetzen ließen, wie fließend die Übergänge zu anderen Verbildlichungs-Strategien waren und welche Herausforderungen dies an eine kritische Überprüfung der heutigen Seherwartungen stellt, soll abschließend ein vor 1510 entstandenes Porträt Kaiser Maximilians I. von der Hand des Joos van Cleve vorführen (Abb. 27). ${ }^{61}$ Noch jüngst glaubte man im Hinblick auf dieses und andere Porträts des Joos von Cleve konstatieren zu dürfen, „dass die politische Dimension der Porträtkunst nicht gerade die Stärke des Künstlers war. "62 Dennoch belegen die zahlreichen Kopien und Varianten zweifelsfrei, daß für die Zeitgenossen offenbar gerade dieses das offizielle oder jedenfalls eines der geläufigsten Konterfeis des Kaisers zumindest in den Niederlanden war (neben den Bildnisprägungen des Bernhard Strigel im restlichen Reich). Dessen Typus fand selbst noch nach 1518, als das Kaiser-Bildnis Dürers entstand (das bei Margarete von Österreich aber offenbar wenig Zustimmung fand), als Vorlage für den Statutenband des Ordens vom Goldenen Vlies Verwendung. Ausgetauscht wurde nur das Objekt in der Hand des Kaisers: Anstatt der ursprünglichen Nelke findet sich in den Varianten ein Schriftstück, selten ein Ring (Abb. 28).

Mit großer Wahrscheinlichkeit wurden diese Varianten zunächst für die seit 1507 installierte Statthalterin der Niederlande, Maximilians Tochter Margarete, gefertigt. 1524 werden im Inventar ihres Besitzes jedenfalls zwei kleine Porträttafeln beschrieben, bei denen es sich um die vorgestellten Gemälde handeln dürfte: „Item ung aultre tableau de la portraiture de l'empereur Maximilien tenant deux fleurs d'ulletz en sa main, habillé de drap d'or, portant la thoison“, und „Aultre portraiture de l'empereur Maximilien, pere de Madame, que dieu pardoint, habillé d'une robbe de drapt d'or fourée de martre, a ung bonnet noir avec le colier de la

59 Zit. Musculus 1556, fol. Biir; Montaigne 1998, Bd. 3, 119f. (III/5, Über einige Verse des Vergil'). - Zur Geschichte etwa Lüttenberg 2005.

60 Flemming 1998.

61 Hand 2004, 20 und 112-114 (Kat. 2.0-2.10); vgl. auch Scailliérez 1991, 84-88, und zuletzt Schütz 2012.

62 So die Schlußfolgerung in dem wichtigen Beitrag von Scailliérez 2011, 104. 
thoison d'or, tenant ung rolet a sa main dextre. "63 Maximilians Reise durch Deutschland in die Niederlande 1507/08 wäre ein naheliegender Entstehungsanlaß gewesen. In der offiziellen Porträtgalerie Margaretes war Maximilian mit Schriftrolle zu sehen. In einem ,privaten' Kabinett der Herrscherin war ihr Vater mit zwei roten Nelken in der Hand dargestellt. In anderen Räumen des Palastes hingen im übrigen weitere Bildnisse des Kaisers, nämlich zwei Versionen des ganz anders angelegten, hieratischen Kaiserporträts von Bernhard Strigel - einmal mit Rüstung und Herrschaftszeichen, einmal mit Schaube, Barett und Schriftstück. ${ }^{64}$ Die Tochter Margarete präsentierte also ein breites Spektrum von vier Darstellungsmöglichkeiten des Herrschers - und dies sicher in Abstimmung mit ihrem Vater. Denn auch wenn Maximilian I. natürlich nicht jedes seiner Bildnisse selbst autorisierte, so war er doch der erste europäische Herrscher, dessen Leben von zarter Kindheit bis zum Totenbett anhand einer bis dato ganz ungewöhnlich dichten Reihe von Bildnissen begleitet wurde. ${ }^{65}$

Am meisten überrascht die heutige Forschung das Bildnis mit Nelke. Der Kaiser ist als junger Mann in eng gewähltem Brustausschnitt zu sehen, eingepaßt in einen Rahmen mit rundem oberem Abschluß, wie es auch von anderen Herrscherporträts des beginnenden 16. Jahrhunderts im Norden geläufig war. Das Gesicht entspricht aber kaum einem knapp 50-jährigen Mann. Die Nelke dagegen findet sich bevorzugt auf Porträts, die im Zusammenhang mit Brautwerbung und Hochzeit stehen. Wenn es in den Jahren um 1500 eine europaweit gültige Porträtkonvention gab, dann dieses Symbol. So hält der englische König Heinrich VII. auf seinem Porträt, das laut Inschrift am 29. Oktober 1505 für Hermann Rinck, einen Agenten Maximilians, gemalt wurde und wohl im Zusammenhang einer (letztlich gescheiterten) Hochzeitswerbung zu sehen ist, ebenfalls eine Nelke - und mehr noch: Dieses Porträt befand sich ebenfalls in der Sammlung der Margarete von Österreich. Verweisen ließe sich auch auf Bartholomäus Zeitbloms Werbebild ebenfalls aus den Jahren um 1505 oder auf das Selbstbildnis des Malers Joos van Cleve im Vorfeld seiner Hochzeit 1519. ${ }^{66}$

Allein bei Maximilian war um 1508/09 kein Verlöbnis in Sicht: Er hatte seine erste Frau, Maria von Burgund, 1477 geehelicht. Nach deren frühem Tod hatte er sich 1494 mit Bianca Maria Sforza verbunden und war also zur Entstehungszeit von Joos van Cleves, Porträt als Brautwerbernder` seit 15 Jahren verheiratet. Zuletzt hat Dagmar Eichberger mit den besten Argumenten die Möglichkeit diskutiert, daß Maximilian hier retrospektiv dargestellt wurde - so wie auch auf seinem sogenannten Hochzeitsguldiner aus den Jahren um 1511, bei dem eine ursprünglich 1477 von Giovanni Candida gefertigte Medaille auf Maximilians Hochzeit mit

63 Zimerman 1885, CI (no. 214 [95]) und XCV (no. 25 [79]). - Ein Porträttyp Maximilians von Bernhard Strigel hat zwar ebenfalls ein Schriftstück in der rechten Hand, aber eine rote, keine schwarze Kopfbedeckung.

64 Beaven/Eichberger 1995; Eichberger 2002, 372-381; zur Frage der Typisierung Müller 2009.

65 Zusammenfassend Metzger 2011; vgl. auch Silver 2008, allerdings ohne Hinweis auf die Porträts des Joos van Cleve.

66 Borchert/Jonckheere 2015, 108f. (Kat. 11). 
Maria von Burgund nochmals als Prägemedaille mit kleinen Veränderungen und der Jahreszahl 1479 aufgelegt wurde. ${ }^{67}$ Deutlich wird allein schon an diesem Vergleichsbeispiel auch, daß kaum die wenig standesgemäße ,Geld-Ehe mit Bianca Maria Sforza, sondern nur die Verbindung mit Maria von Burgund als Erinnerung in Frage kommt - und daß der Umstand, daß Maximilian erst 1478, also nach der ersten Hochzeit, in den Orden vom Goldenen Vlies aufgenommen wurde, in dieser retrospektiven Konstruktion kein Problem darstellte. Die politische Intention und Botschaft dabei dürfte gewesen sein, die Legitimation der Tochter Margarete als Statthalterin der (Burgundischen) Niederlande zu bekräftigen.

$\mathrm{Zu}$ fragen bleibt allerdings weiterhin, ob diese spezifische Absicht allein (oder auch der Hinweis auf kontext- und orts-spezifische Ausformungen der Bildnisse Maximilians, die in den Niederlanden eben anders aussahen als im Südosten des Reiches) die weite Verbreitung dieses Bildnisses wirklich erklären kann. Zumal die verschiedenen späteren Bildversionen, an deren Produktion dann auch noch eine andere, die Werkstatt des Meisters von Frankfurt beteiligt war, nur ausnahmsweise den Eindruck vermitteln, hier solle Maximilian als junger Mann rückblickend evoziert werden. ${ }^{68}$ Die anderen Porträts zeigen den Dargestellten in gesetzterem Alter ohne hier darüber diskutieren zu wollen, ob er wirklich als 50-jähriger erscheint, der er 1509 gewesen sein müßte. Der entscheidende Punkt ist vielmehr: So wie Maximilian auf Porträts als Herrscher in Rüstung, so wie er als antikischer Kaiser im Profil oder aber als guter, gelehrter und gerechter Regent mit Brief und in Redegestus auftreten kann, so scheint es auch Sinn zu machen, daß Maximilian als Liebender und Werbender auftritt - und zwar unabhängig von einer konkreten historischen Situation. Demonstriert werden soll die Attraktivität des Herrschers, wie sie sich in dem jugendlichen Liebeswerbenden zeigt (und offenbar als Bildnistypus erhalten blieb, auch wenn dann ein gealtertes Herrscherantlitz wiedergegeben wurde); das Liebeswerben kann zudem als Sinnbild für die Frieden und Einheit stiftenden Qualitäten des Kaisers verstanden werden; und es geht darum, durch Liebeswerben und anziehend(-jugendlich)en Herrscherkörper die überragende ,Höfischkeit' oder Courtoisie des Kaisers zu signalisieren. Das Bildnis Maximilians ruft einen Verhaltens-Codex auf, der sowohl für den, vollkommenen Ritter ${ }^{`}$ im Frankreich des 15. Jahrhunderts als auch für den ,vollkommenen Höfling Italiens, wie ihn etwa Baldassare Castigliones Cortegiano kodifizierte, galt: Er mußte ,in Liebe' sein - überhaupt erst die Liebe (und zwar nicht nur im Hinblick auf Frauen, sondern auch auf Freunde) ermöglichte ihnen ihre volle Entfaltung als tugendhafte und soziale Wesen. ${ }^{69}$ Als anzügliche Zuspitzung dieser Denkweise darf noch Pierre de Brantômes späterer Skandalbericht vom französischen Hof gel-

67 Winter 2013, 64-86.

68 Goddard 1984, 132, Nr. 8-10.

69 Vgl. etwa Le livre des faits de Jacques de Lalaing, in: Chastellain 1886, 15; Freigang 2005; zu Castiglione und dem italienischen Diskurs zusammenfassend Kolsky 1991, 146f.; zum größeren Kontext Jaeger 1999, 198-200. 
ten, in dem von Franz I. kolportiert wird, dieser habe alle Höflinge ohne Maitressen für „Idioten“ gehalten. ${ }^{70}$

Ein solches Verständnis würde jedenfalls auch andere Monumente von Maximilians (Selbst-)Darstellung besser zu verstehen erlauben: Die Malereien und Reliefs des auf "1500" datierten Goldenen Dachls in Innsbruck gehören in einen sehr spezifischen, noch immer nicht restlos ausgedeuteten Kontext, zeigen aber jedenfalls den Kaiser als jungen Liebhaber und möglicherweise Liebesnarren. ${ }^{71}$ Im Theuerdank, der wohl unmittelbar im Anschluß an die Niederlandefahrt und das Porträt des Joos van Cleve um 1510 zu konzipieren begonnen wurde, thematisiert Maximilian in der Verkleidung eines fiktiven mittelalterlichen Ritter-Abenteuers seine Brautfahrt zu Maria von Burgund. ${ }^{72}$ Auch wenn das literarische ,Brautwerbungsschema' in der Durchführung gegenüber anderen Themen: dem Meistern gefährlicher Situationen, der Auseinandersetzung mit den niederländischen Provinzen und dem Projekt eines Türkenkreuzzugs, nicht dominiert, bleibt es als Grundmotiv doch stets präsent. Und so gilt auch in diesem Fall, daß 1517, als das Werk im Druck erschien, erneut ein jugendlicher Liebender und Liebeswerbender als alter ego des alternden Kaisers diente.

Angesichts des hier skizzierten Spektrums an Selbst- und Fremddarstellungen ,erotischer Machtausübung' wäre eigentlich zu erwarten, daß diese Visualisierungsmöglichkeiten umgehend auch negativ für Diffamierung und Gegenpropaganda eingesetzt wurden. Zumal in Texten politische Gegner seit jeher mit Vorwürfen von Impotenz oder sexueller Devianz - unter dem Stichwort ,Sodomie' zusammengefaßt - überzogen wurden. In der Tat gibt es Einzelfälle: Sieht man von den sexualisierten, transgressiven Randillustrationen mittelalterlicher Handschriften ab (die freilich eher eine generelle Moral- und Sozialkritik formulieren), ließe sich auf die mit der Reformation öfter dargestellte Idee von Papst und Kirche als ,Hure Babylon', auf den oben angeführten Maiolika-Tellers mit dem Sturz Franz' I. oder auf eine Medaille des Hunnenkönigs Attila mit Phalluskopf-Revers verweisen. ${ }^{73}$ Weitere Verbreitung scheint die sexualisierte Bild-Satire auf den Herrscher aber erst mit dem 17. Jahrhundert zu gewinnen. So werden etwa in Les Hermaphrodites, einem anonym und ohne Angabe zu Ort und Zeit 1605 in Paris erschienen und wohl von Thomas Artus verfaßten Büchlein, unter der Fiktion einer Insel der Hermaphroditen die zeitgenössischen Zustände in Frankreich kritisiert. Dabei stehen die ambiguen und im späten 16. Jahrhundert intensiv diskutierten ,GeschlechtsMonster für alles vermeintlich Widernatürliche und Untugendhafte, beginnend mit den homoerotischen Liebesverhältnissen Heinrichs III. zu seinen mignons. ${ }^{74}$

70 Brantôme 1960, $297 f$.

71 Pokorny 2002/2003.

72 Müller 1982, v. a. 109-112; Müller/Ziegeler 2015; zur Tradition vgl. Schmid-Cadalbert 1985.

73 Camille 1992; Waddington 2004.

74 Artus 1996; Pierre de l'Etoile kommentiert in seinem Journal zum 11. April 1605: „Ce petit libelle (qui étoit assez bien fait), sous le nom de cette isle imaginaire, découvroit les mœurs et façons de faire impies et vicieuses de la cour, faisant clairement voir clairement que la France est maintenant 
Das gestochene Titelblatt (das offenbar auch in einer gleichzeitigen Holzschnittvariante existiert) zeigt einen modisch gekleideten Adligen, dessen Kopf in den Gesichtszügen, vor allem aber durch Frisur und Schmuck vollkommen, effeminiert ${ }^{\text {` }}$ ist (Abb. 29). Alle Kritik erfolgt nun unter sexualisierten Vorzeichen - angeprangert wird die Verweiblichung der Macht, wo eigentlich männliche Potenz agieren müßte. Sehr deutlich wird aber auch, daß das eigentliche Potential einer sexualisierten, teils dann geradezu pornografischen Bildkritik an den Mächtigen und an den Machtverhältnissen damit noch lange nicht ausgeschöpft war, wie die späteren Beispiele seit dem 18. Jahrhundert belegen. ${ }^{75}$

\section{Bibliographie}

\section{Quellen}

Artus, Thomas (1996): Lisle des hermaphrodites. Hrsg. von Claude-Gilbert Dubois. Genf: Droz (= Textes littéraires français, 467).

Benedetti, Alessandro (1549): Il fatto d'arme del Tarro fra i principi italiani et Carlo ottavo Re di Francia. Venedig: Giolito de Ferrara.

Bologna, Giulia (Hrsg.) (1989): Tutte le dame del re. Ritratti di dame milanesi per Francesco $I$, re di Francia. Mailand: Biblioteca Trivulziana.

Brantôme, Pierre de Bourdeille, seigneur de ([1655] 1960): Les Dames galantes. Hrsg. von Maurice Rat. Paris: Garnier.

Calepino, Ambrogio (1570): Dictionarium. Paris: Macé.

Chastellain, Georges (1886): Euvres. Bd. 8. Hrsg. von Kervyn de Lettehove. Brüssel: Heussner.

Corio, Bernardino (1503): Historia continente da l'origine di Milano tutti li gesti, fatti, e detti preclari, ... . Mailand: Minuziano.

Dürer, Albrecht (1956): Schriftlicher Nachlaß. Bd. 1. Hrsg. von Hans Rupprich. Berlin: Deutscher Verein für Kunstwissenschaft.

Dupuys, Remy (1515): La tryumphante et solemnelle entree faicte sur le nouuel et ioyeux aduenement de ... Monsieur Charles prince des hespaignes Archiduc daustrice duc de bourgongne Co[n]te de Flandres etc., Paris [Nachdruck hrsg. und eingef. von Sydney Anglo, Amsterdam/New York 1973].

Filarete, Antonio Averlino gen. (1890): Tractat über die Baukunst. Hrsg. von Wolfgang von Oettingen. Wien: Graeser (= Quellenschriften für Kunstgeschichte und Kunsttechnik des Mittelalters und der Neuzeit, 3).

Hobbes, Thomas (1996): Leviathan. Hrsg. von Iring Fetscher, übers. von Walter Euchner. Frankfurt am Main: Suhrkamp (= stw, 462).

le repaire et l'asyle de tout vice, volupté et impudence au lieu que jadis elle étoit une académie honorable et un séminaire de vertu. Le Roy le voulut voir, et se fit lire ; et encore qu'il le trouvât un peu libre et trop hardi, il se contenta néanmoins d'en apprendre le nom de l'auteur, qui étoit Artus Thomas, lequel, il ne voulut qu'on recherchât, faisant conscience, disoit-il, de fâcher un homme pour avoir dit la vérité." - Dazu etwa Cady 1996; Long 2006; Ferguson 2008; Poirier 2010 .

75 Vgl. für Beispiele des mittleren 17. Jahrhunderts etwa Bredekamp 2006, 49-51 (zu einem Stich des Abraham Bosse mit melancholischem König), und Kahn 1907, Beilage nach 64; für die Zeit ab ca. 1700 etwa Ziegler 2010, 163-167; Gatrell 2006. 
Luzio, Alessandro/Renier, Rodolfo (1890): „Delle relazioni di Isabella d'Este Gonzaga con Ludovico e Beatrice Sforza“, in: Archivio storico lombardo, 2 ser. 7, 74-119, 346-399 und 619-674.

Machiavelli, Niccolò (2013): Il Principe. Hrsg. von Gabriele Pedullà. Rom: Donzelli.

Malipiero, Domenico (1843): Annali veneti dall'anno 1457-1500. Florenz: Vieusseux.

Mirandula, Octavianus (1544): Illustrium poetarum flores. Straßburg: Rihelius.

Montaigne, Michel de (1998): Essais. Übers. von Hans Stilett. Frankfurt am Main: Eichborn.

Musculus, Andreas (1556): Vom Hosen Teuffel. Frankfurt an der Oder: Eichorn.

Saavedra Fajardo, Diego de (1655): Ein Abriss eines Christlich-Politischen Printzens / in CI. Sinn-bildern und mercklichen Symbolischen Sprüchen. Amsterdam.

Valladier, André (1600): Labyrinthe royal de l'Hercule gaulois triomphant. Avignon: Bramereau.

Vasari, Giorgio (1987): Le vite de' più eccellenti pittori, scultori e architettori nelle redazioni del 1550 e 1568. Bd. 6. Hrsg. von Rosanna Bettarini, komm. von Paola Barocchi. Florenz: Sansoni.

Vocabolario degli Accademici della Crusca (1612). Venedig: Giovanni Alberti.

Zimerman, Heinrich (1885): „Quellen zur Geschichte der kaiserlichen Haussammlung und der Kunstbestrebungen des allerdurchlauchtigsten Erzhauses“, in: Jahrbuch der kunsthistorischen Sammlungen des allerhöchsten Kaiserhauses 3.

\section{Forschungsliteratur}

Ames-Lewis, Francis/Rogers, Mary (Hrsg.) (1998): Concepts of Beauty in Renaissance Art. Aldershot: Ashgate.

Antonovics, A.V. (1995): „Il semble que se soit là un vrai Paradis terrestre': Charles VIII's conquest of Naples and the French Renaissance“, in: Abulafia, David (Hrsg.): The French Descent into Renaissance Italy. 1494-95. Antecedents and Effects. Aldershot: Variorum, 311-325.

Attwood, Philip (2004): „Cellini's coins and medals“, in: Gallucci, Margaret A./Rossi, Paolo L. (Hrsg.): Benvenuto Cellini. Sculptor, goldsmith, writer. Cambridge: Cambridge University Press, 97-120.

Baader, Hannah (1999): „Giovanni Paolo Lomazzo: Das Porträt als Zeichensystem (1584)“, in: Preimesberger, Rudolf/Bader, Hannah/Suthor, Nicola (Hrsg.): Porträt. Berlin: Reimer, 307-315.

Baxandall, Michael (1971): Giotto and the Orators. Humanist Observers of Painting in Italy and the Discovery of Pictorial Composition. 1350-1450. Oxford/New York: Clarendon Press/Oxford University Press (= Oxford Warburg Studies).

Beaven, Lisa/Eichberger, Dagmar (1995): „Family Members and Political Allies: The Portrait Gallery of Margaret of Austria in Mechelen", in: Art Bulletin 77, 225-248.

Bertelli, Sergio (1995): Il corpo del re. Sacralità del potere nell' Europa medievale e moderna. Florenz: Ponte alle Grazie [zuerst 1990].

Bischoff, Cordula (1998): „Die Schwäche des starken Geschlechts. Herkules und Omphale und die Liebe in bildlichen Darstellungen des 16. bis 18. Jahrhunderts", in: Dinges, Martin (Hrsg.): Hausväter, Priester, Kastraten. Zur Konstruktion von Männlichkeit in Spätmittelalter und Früher Neuzeit. Göttingen: Vandenhoeck \& Ruprecht (= Sammlung Vandenhoeck), 153-181.

Borchert, Till-Holger/Jonckheere, Koenraad (Hrsg.) (2015): Portraits de la Renaissance aux Pays-Bas. Brüssel: Uitgeverij Hannibal.

Borggrefe, Heiner (2006): „Tizians ruhende Göttinnen und die Dienerinnen der Liebe“, in: Tacke, Andreas (Hrsg.): „... wir wollen der Liebe Raum geben“. Konkubinate geistlicher 
und weltlicher Fürsten um 1500. Vorträge der III. Moritzburg-Tagung (Halle/Saale) vom 31. März bis 2. April 2006. Göttingen: Wallstein, 393-421.

Bredekamp, Horst (32006): Thomas Hobbes. Der Leviathan. Das Urbild des modernen Staates und seine Gegenbilder. 1651-2001. Berlin: Akademie.

Bredekamp, Horst (2014): Der schwimmende Souverän. Karl der Große und die Bildpolitik des Körpers. Eine Studie zum schematischen Bildakt. Berlin: Wagenbach (= Kleine kulturwissenschaftliche Bibliothek, 86).

Brown, Cynthia (2011): The Queen's Library: Image-Making at the Court of Anne of Brittany, 1477-1514. Philadelphia: University of Pennsylvania Press (= Material Texts).

Bulst, Wolfger (2013): „Hercules Estensis“, in: Weigel, Thomas/Poeschke, Joachim (Hrsg.): Leitbild Tugend. Die Virtus-Darstellungen in italienischen Kommunalpalästen und Fürstenresidenzen des 14. bis 16. Jahrhunderts. Münster: Rhema (= Symbolische Kommunikation und gesellschaftliche Wertesysteme, 36), 97-150.

Cady, Joseph (1996): „The ,Masculine Love' of the ,Princes of Sodom': Practising the Art of Ganymede" at Henri III's Court. The Homosexuality of Henri III and His Mignons in Pierre de L'Estoile's Mémoires-Journaux“, in: Murray, Jacqueline/Eisenbichler, Konrad (Hrsg.): Desire and Discipline. Sex and Sexuality in the Premodern West. Toronto: University of Toronto Press, 123-154.

Camille, Michael (1992): Image on the Edge. The margins of medieval art. Cambridge, Mass.: Harvard University Press (= Essays in art and culture).

Campbell, C. Jean (1997): The Game of Courting and the Art of the Comune of San Gimignano 1290-1320. Princeton: Princeton University Press.

Campbell, Lorne (1990): Renaissance Portraits. European Portrait-Painting in the 14th, 15th and 16th Centuries. New Haven/London: Yale University Press.

Cauchies, Jean-Marie (1998): „La signification politique des entrées princières dans les Pays-Bas: Maximilien d'Autriche et Philippe le Beau“, in: ders. (Hrsg.): $A$ la Cour de Bourgogne: Le duc, son entourage, son train. Turnhout: Brepols, 137-152.

Civai, Mauro (Hrsg.) (2008): ,Continenza' di Scipione. Il tema della , magnanimitas' nell'arte italiana. Siena: Protagon.

Contardi, Bruno/Lilius, Henrik (Hrsg.) (1984): Quando gli dei si spogliano. Il bagno di Clemente VII a Castel Sant'Angelo e le altre stufe romane del primo Cinquecento. Rom: Romana societa (= Cataloghi del Museo Nazionale di Castel Sant'Angelo, 2).

Coppel Aréizaga, Rosario (1998): Museo del Prado - Catálogo de la escultura de época moderna. Siglos XVI-XVIII. Madrid: Museo del Prado.

Couderc, Camille (1896): „L'entrée solennelle de Louis XI à Paris (31 août 1461)“, in: Mémoires de la Société de l'Histoire de Paris et de l'Île-de-France 23, 125-166.

Crawford, Katherine (2010): The Sexual Culture of the French Renaissance. Cambridge/New York: Cambridge University Press.

Cropper, Elizabeth (1995): „The Place of Beauty in the High Renaissance and its Displacement in the History of Art", in: Vos, Alvin (Hrsg.): Place and Displacement in the Renaissance. Binghampton, NY: Centre for Medieval and Early Renaissance Studies, State University of New York (= Medieval and Renaissance texts and studies, 132), 159205.

Denis, Anne (1979): Charles VIII et les Italiens: histoire et mythe. Genf: Droz.

Eichberger, Dagmar (2002): Leben mit Kunst-Wirken durch Kunst. Sammelwesen und Hofkunst unter Margarete von Österreich, Regentin der Niederlande. Turnhout: Brepols.

Eschenfelder, Chantal (1991): Die Bäder Franz I. in Fontainebleau. München: tuduv (= Schriften aus dem Institut für Kunstgeschichte der Universität München, 55).

Fabiański, Marcin (1998): Correggio’s Erotic Poesie. Krakau: Instytut Historii Sztuki Uniwersytetu Jagiellońskiego. 
Fenichel Pitkin, Hanna (1984): Fortune Is a Woman. Gender and Politics in the Thought of Niccolò Machiavelli. Berkeley: University of California Press.

Ferguson, Gary (2008): Queer (re)readings in the French Renaissance: homosexuality, gender, culture, Aldershot: Ashgate.

Filzmoser, Romana (2014): Hurenbilder. Ein Motiv in der Druckgraphik des 17. und 18. Jahrhunderts. Köln/Weimar/Wien: Böhlau (= Studien zur Kunst, 27).

Findlen, Paula (1994): „Humanismus, Politik und Pornographie im Italien der Renaissance“, in: Hunt, Lynn (Hrsg.): Die Erfindung der Pornographie. Obszönität und die Ursprünge der Moderne. Frankfurt am Main: Fischer Taschenbuch Verlag, 44-114.

Flemming, Victoria von (1998): „Die nackten Toten - Caterina de’ Medici und die Diskursivierung der Körper", in: Wunder, Heide/Engel, Gisela (Hrsg.): Geschlechterperspektiven. Forschungen zur Frühen Neuzeit. Königstein: Helmer, 408-424.

Foucault, Michel (1976-1984): Histoire de la sexualité. 3 Bde. Paris: Gallimard.

Freedberg, David (1989): The Power of Images. Studies in the history and theory of response. Chicago: University of Chicago Press.

Freigang, Christian (2005): „,Fantaisie et ymaginacion': Selbstreflexion von Höfischkeit am provençalischen Hof unter René I.“, in: ders./Schmitt, Jean-Claude (Hrsg.): Hofkultur in Frankreich und Europa im Spätmittelalter. Berlin: Akademie (= Passagen), 209-243.

Fritz, Michael P. (2002): „,pieno d'una certa argutia gioconda et sottile ...' Kardinal Bibbiena und die hohe Kunst der Diplomatie“, in: Tewes, Götz-Rüdiger/Rohlmann, Michael (Hrsg.): Der Medici-Papst Leo X. und Frankreich. Tübingen: Mohr Siebeck (= Spätmittelalter und Reformation, Neue Reihe, 19), 427-467.

Gatrell, Vic A.C. (2006): City of Laughter. Sex and Satire in Eighteenth-Century London. London: Atlantic.

Goddard, Stephen H. (1984): The Master of Frankfurt and his Shop. Brüssel: AWLSK (= Verhandelingen van de Koninklijke Academie voor Wetenschappen, Letteren en Schone Kunsten van België, Klasse der Schone Kunsten, 38).

Halleux, Élisa de (2012): „L'androgynie d'Hercule, entre dérision et glorification du prince“, in: Morel, Philippe (Hrsg.): Le miroir et l'espace du prince dans l'art italien de la Renaissance. Tours: Presses universitaires François Rabelais de Tours, 145-164.

Hand, John O. (2004): Joos van Cleve. The Complete Paintings. New Haven/London: Yale University Press.

Haug, Walter (1989): „Huge Scheppel - der sexbesessene Metzger auf dem Lilienthron“, in: Heinzle, Joachim/Johnson, L. Peter/Vollmann-Profe, Gisela (Hrsg.): Wolfram-Studien XI. Chansons de geste in Deutschland. Schweinfurter Kolloquium 1988. Berlin: Schmidt, 185-205.

Himmelmann, Nikolaus (1985): Ideale Nacktheit. Opladen: Westdeutscher Verlag.

Hochner, Nicole (2006): Louis XI. Les dérèglements de l'image royale (1498-1515). Seyssel: Champ Vallon (= Époques).

Hunt, Lynn (1989): Symbole der Macht - Macht der Symbole. Die Französische Revolution und der Entwurf einer politischen Kultur. Frankfurt am Main: Fischer.

Jaeger, C. Stephen (1999): Ennobling Love. In Search of a Lost Sensibility. Philadelphia: University of Pennsylvania Press.

Jestaz, Bertrand (1989): „Le reliquiaire de Charles V. perdu par Charles VIII a Fornoue“, in: Bulletin Monumental 147, 7-10.

Jollet, Étienne (2006): „L'œil du prince: le regard dans les portraits royaux par Jean et François Clouet", in: Gaehtgens, Thomas W./Hochner, Nicole (Hrsg.): L'image du roi de François $I^{e r}$ à Louis XIV. Paris: Maison des sciences de l'homme (= Passagen, 10), 343358.

Kahn, Gustave (1907): Das Weib in der Karikatur Frankreichs. Stuttgart: Schmidt. 
Knuth, Michael (2007): „I Paggi del Monumento Vendramin nel museo di Berlino: storia e stato di conservazione", in: Ceriana, Matteo (Hrsg.): Tullio Lombardo, scultore e architetto nella Venezia del rinascimento. Verona: Cierre, 15-22.

Kolsky, Stephen (1991): Mario Equicola. The real courtier. Genf: Droz.

Koos, Marianne (2006): Bildnisse des Begehrens. Das lyrische Männerporträt in der venezianischen Malerei des frühen 16. Jahrhunderts. Giorgione, Tizian und ihr Umkreis. Emsdetten/ Berlin: Imorde.

Krystof, Doris (1997): Werben für die Kunst. Bildliche Kunsttheorie und das Rhetorische in den Kupferstichen von Hendrick Goltzius. Hildesheim u.a.: Olms (= Studien zur Kunstgeschichte, 107).

Lavin, Irving (1993): Past-Present. Essays on Historicism in Art from Donatello to Picasso, Berkley u.a.: University of California Press (= Studies on the History of Society and Culture/Una's Lectures, 6).

Legaré, Anne-Marie (2011): „Joanna of Castile's Entry into Brussels: Viragos, Wise and Virtuous Women“, in: Green, Karen/Mews, Constant J. (Hrsg.): Virtue Ethics for Women 1250-1500. Heidelberg/London/New York: Springer, 177-186.

Long, Kathleen P. (2006): Hermaphrodites in Renaissance Europe. Aldershot: Ashgate.

Lüttenberg, Thomas (2005): „The Cod-piece - A Renaissance Fashion between Sign and Artefact", in: The Medieval History Journal 8, 49-81.

Markham Schulz, Ann (2014): The Sculpture of Tullio Lombardo, London/Turnhout: Brepols.

Marti, Susan/Borchert, Till-Holger/Keck, Gabriele (Hrsg.) (2008): Karl der Kühne (14331477). Kunst, Krieg und Hofkultur. Stuttgart/Bern/Brügge: Belser/Mercatorfonds.

Martini, Mario (1982): Bernardino Cacciante Aletrinate. Contributo alla storia dell'umanesimo. Sora: Centro di Studi Sorani, Vincenzo Patriarca' (= Poeti e scrittori di ciociaria).

Matthews Grieco, Sara (1991): Ange ou diablesse: le représentations de la femme au XVT siècle. Paris: Flammarion (= Histoires).

Melzer, Christien (2014): Virtuos imitiert. Die Meisterstiche des Hendrick Goltzius (15581617). Bremen: Kunstverein Bremen (= Kataloge des Kupferstichkabinetts, 7).

Metzger, Christof (2011): „Eine Glaubensfrage. Auf der Suche nach der Wahrheit im Bildnis der Dürerzeit", in: Haag, Sabine/Lange, Christine/Metzger, Christof/Schütz, Karl (Hrsg.): Dürer. Cranach. Holbein. Die Entdeckung des Menschen: Das deutsche Porträt um 1500. Wien/München: Hirmer, 21-26.

Müller, Jan-Dirk (1982): Gedechtnus. Literatur und Hofgesellschaft um Maximilian I. München: Fink (= Forschungen zur Geschichte der älteren deutschen Literatur, 2).

Müller, Jan-Dirk/Ziegeler, Hans-Joachim (Hrsg.) (2015): Maximilians Ruhmeswerk. Künste und Wissenschaften im Umkreis Kaiser Maximilians I. Berlin/Boston: De Gruyter.

Müller, Marcus (1999): „Der neuerliche Angriff auf die Minneburg. Sprachbild und Bildsprache der Minne", in: Vavra, Elisabeth (Hrsg.): Bild und Abbild vom Menschen im Mittelalter. Akten der Akademie Friesach "Stadt und Kultur im Mittelalter", Friesach (Kärnten), 9.-13. September 1998. Klagenfurt: Wieser, 89-109.

Müller, Matthias (2009): „Die Individualität des Fürsten als Illusion der Malerei. Zum Verhältnis von Individualität, Typus und Schema in Regentenporträts der beginnenden Frühen Neuzeit", in: Auge, Oliver/Werlich, Ralf-Gunnar/Zeilinger, Gabriel (Hrsg.): Fürsten an der Zeitenwende zwischen Gruppenbild und Individualität. Ostfildern: Thorbecke, 103-127.

Nagle, Jean (1998): La civilization du cour. Histoire du sentiment politique en France du XII au XIX $X^{e}$ siècle. Paris: Fayard.

Panofsky, Erwin (1930): Hercules am Scheidewege und andere antike Bildstoffe in der neuen Kunst. Leipzig/Berlin: Teubner (= Studien der Bibliothek Warburg, 18).

Paravicini Bagliani, Agostino (1997): Der Leib des Papstes. Eine Theologie der Hinfälligkeit. München: C. H. Beck [zuerst ital. 1994]. 
Paravicini Bagliani, Agostino (Hrsg.) (2014): Le corps du prince. Florenz: SISMEL/Edizioni del Galluzzo (= Micrologus, 22).

Pfisterer, Ulrich (2014): Kunst-Geburten. Kreativität, Erotik, Körper in der Frühen Neuzeit. Berlin: Wagenbach (= Kleine kulturwissenschaftliche Bibliothek, 87).

Philipp, Marion (2011): Ehrenpforten für Kaiser Karl V. Festdekorationen als Medien politischer Kommunikation. Münster: Lit (= Kunstgeschichte, 90).

Poeschke, Joachim (1992): Die Skulptur der Renaissance in Italien. Bd. 2. Michelangelo und seine Zeit. München: Hirmer.

Poirier, Guy (2010): Henri III de France en mascerades imaginaires. Mours, humeurs et comportements d'un roi de la Renaissance. Québec: Presses de l'Université Laval.

Pokorny, Erwin (2002/2003): „Minne und Torheit unter dem Goldenen Dachl. Zur Ikonographie des Prunkerkers Maximilians I. in Innsbruck", in: Jahrbuch des Kunsthistorischen Museums Wien 4/5, 31-46.

Prohaska, Wolfgang/Ferino-Pagden, Sylvia/Wied, Alexander (Hrsg.) (1995): Eros und Mythos. Kunst am Hof Rudolfs II. Wien: Kunsthistorisches Museum.

Ramsey, Ann W. (2007): „The ritual meaning of Henri IV's 1594 Parisian entry“, in: Russell, Nicolas/Visentin, Hélène (Hrsg.): French Ceremonial Entries in the Sixteenth Century. Event, Image, Text. Toronto: Centre for Reformation and Renaissance Studies (= Essays and studies, 11), 189-205.

Rank, Otto (1914): „Um Städte werben“, in: Internationale Zeitschrift für ärztliche Psychoanalyse 2, 50-58.

Reeser, Todd W. (2006): Moderating Masculinity in Early Modern Culture. Chapel Hill: The University of North Carolina Press.

Sandt, Udolpho van de (1999), in: Rosenberg, Pierre (Hrsg.): Dominique-Vivant Denon. L'oil de Napoléon. Paris, Musée du Louvre, 20 octobre 1999-17 janvier 2000. Paris: Réunion des Musées Nationaux, 94f. (Kat. 51).

Santosuosso, Antonio (1994): „Anatomy of Defeat in Renaissance Italy. The Battle of Fornovo in 1495“, in: The International History Review 16, 221-440.

Scailliérez, Cécile (1991): Joos van Cleve au Louvre. Paris: Réunion des Musées Nationaux.

Scailliérez, Cécile (2011): „Die Porträtkunst Joos van Cleves“, in: Brink, Peter van den (Hrsg.): Joos van Cleve. Leonardo des Nordens. Aachen/Stuttgart: Belser, 87-111.

Schmid-Cadalbert, Christian (1985): Der, Ortnit AW' als Brautwerbungsdichtung. Ein Beitrag zum Verständnis mittelhochdeutscher Schemaliteratur. Bern: Francke (= Bibliotheca germanica, 28).

Schneller, Robert W. (1981-82): „Imperial Themes: the period of Charles VIII“, in: Simiolus 12, 5-69.

Schrader, Stephanie (2010): „Gossart's mythological nudes and the shaping of Philip of Burgundy's erotic identiy“, in: Ainsworth, Maryan W./Alsteens, Stijn/Orenstein, Nadine M. (Hrsg.): Man, Myth, and Sensual Pleasures. Jan Gossart's Renaissance. New York: Yale University Press, 57-67.

Schütz, Karl (2012): „Joos van Cleve - Kaiser Maximilian I.“, in: Michel, Eva/ Sternath, Maria L. (Hrsg.): Kaiser Maximilian I. und die Kunst der Dürerzeit. Wien/München: Prestel, 134f. (Kat. 2).

Schulte, Regina (Hrsg.) (2002): Der Körper der Königin. Geschlecht und Herrschaft in der höfischen Welt seit 1500. Frankfurt am Main: Campus.

Silver, Larry (2008): Marketing Maximilian. The Visual ideology of a Holy Roman Emperor. Princeton/Oxford: Princeton University Press.

Spieth, Darius A. (2001): The Printed Work of Vivant Denon (1747-1825). 2 Bde. PhD diss. University of Illinois at Urbana. 
Springer, Carolyn (2010): Armour and Masculinity in the Italian Renaissance. Toronto: University of Toronto Press.

Steigerwald, Jörn (2014): Amors Renaissance. Modellierungen himmlischer und irdischer Liebe in der Literatur des Cinquecento. Wiesbaden: Harrassowitz (= Culturae, 10).

Steinberg, Leo (21996): The Sexuality of Christ in Renaissance Art and in Modern Oblivion. Chicago: University of Chicago Press [zuerst 1983].

Talvacchia, Bette (1999): Taking Positions. On the Erotic in Renaissance Culture. Princeton, NJ: Princeton University Press.

Tauber, Christine (2009): Manierismus und Herrschaftspraxis. Die Kunst der Politik und die Kunstpolitik am Hof von François $I^{\text {er }}$. Berlin: Akademie (= Studien aus dem WarburgHaus, 10).

Theisen, Maria (1999): „Anmerkungen zur Ikonographie des Titelblatts der Goldenen Bulle, Österreichische Nationalbibliothek, Cod. 338“, in: Umeni 47, 266-277.

Tuttle, Richard J. (2015): The Neptune Fountain in Bologna. Bronze, Marble, and Water in the Making of a Papal City. London/Turnhout: Brepols.

Vandenbroek, Paul (2012): „A bride amidst heroines, fools and savages. The Joyous Entry into Brussels by Joanna of Castile, 1496 (Berlin, Kupferstichkabinett, Ms. 78D5)“, in: Jaarboek Koninklijk Museum voor Schone Kunsten Antwerpen, 153-194.

Verheyen, Egon (1977): The Palazzo del Te in Mantova. Images of Love and Politics. Baltimore/London: Johns Hopkins University Press.

Waddington, Raymond B. (2001): „The Bisexual Portrait of Francois I: Fontainebleau, Castiglione, and the Tone of Courtly Mythology“, in: Brink, Jean R. (Hrsg.): Playing with Gender: A Renaissance Pursuit. Urbana: University of Illinois Press, 99-132.

Waddington, Raymond B. (2004): Aretino's Satyr. Sexuality, Satire and Self-projection in Sixteenth-century Literature and Art. Toronto: University of Toronto Press.

Wenzel, Michael (2001): Heldinnengalerien - Schönheitsgalerien. Studien zu Genese und Funktion weiblicher Bildnisgalerien 1470-1715. Dissertation Univ. Heidelberg [online].

Wilson-Chevalier, Kathleen (1993): "Women on Top at Fontainebleau", in: Oxford Art Journal 16, 34-48.

Winter, Heinz (2013): Die Medaillen und Schaumünzen der Kaiser und Könige aus dem Haus Habsburg im Münzkabinett des Kunsthistorischen Museums Wien. Bd. 1: Friedrich III. und Maximilian I. Wien: Verlag der Österreichischen Akademie der Wissenschaften.

Zanger, Abby E. (1997): Scenes form the Marriage of Louis XIV. Nuptial Fictions and the Making of Absolutist Power. Stanford: Stanford University Press.

Zanger, Abby E. (2002): „Was der König im Bauch des Tieres sah oder wie der Löwe in die Königin kam. Allegorien königlicher Fortpflanzung beim Einzug in Lyon 1622", in: Schulte, Regina (Hrsg.): Der Körper der Königin. Geschlecht und Herrschaft in der höfzschen Welt seit 1500. Frankfurt am Main: Campus, 115-134.

Ziegler, Hendrik (2010): Der Sonnenkönig und seine Feinde. Die Bildpropaganda Ludwigs XIV. in der Kritik. Petersberg: Imhof (= Studien zur internationalen Architektur- und Kunstgeschichte, 79).

Ziegler, Joseph (2004): „Médicine et physiognomie du XIV ${ }^{\mathrm{e}}$ au début du XVI ${ }^{\mathrm{e}}$ siècle“, in: Médiévales 46, 89-108.

Zorach, Rebecca (2005): Blood, Milk, Ink, Gold: Abundance and Excess in the French Renaissance. Chicago: University of Chicago Press. 


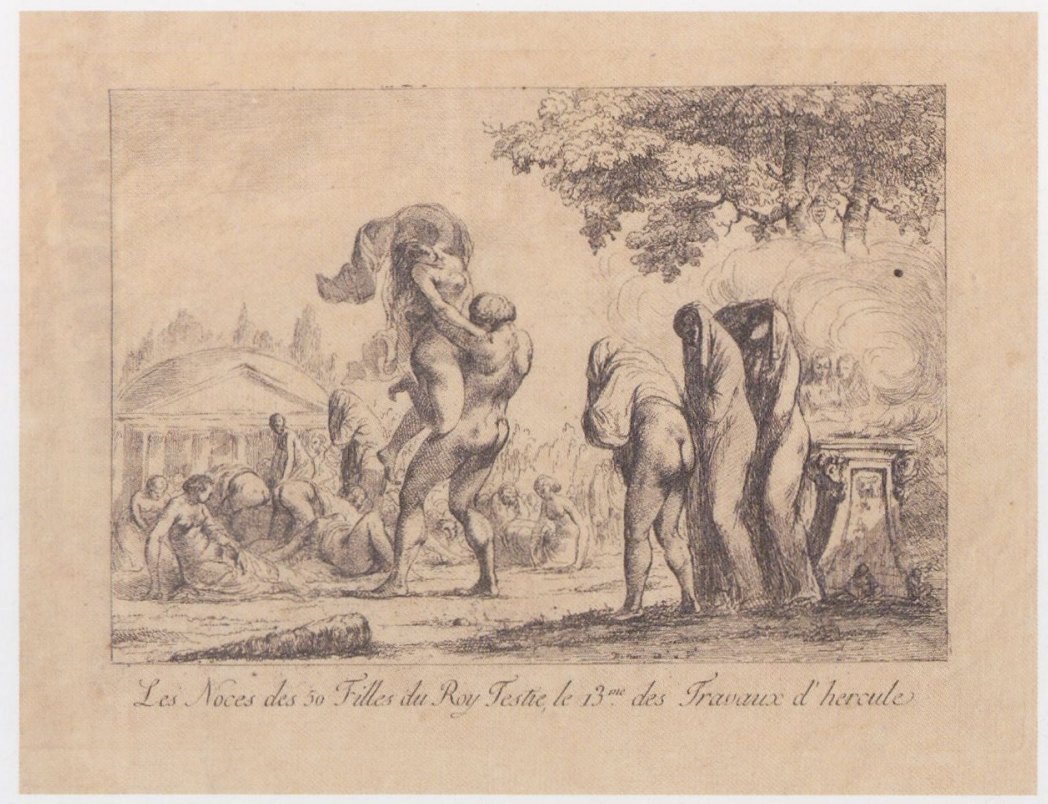

Abbildung 21: Dominique Vivant Denon: Les Noces des 50 Filles du Roy Testic, le 13. ${ }^{\text {me }}$ des Travaux d'hercule.

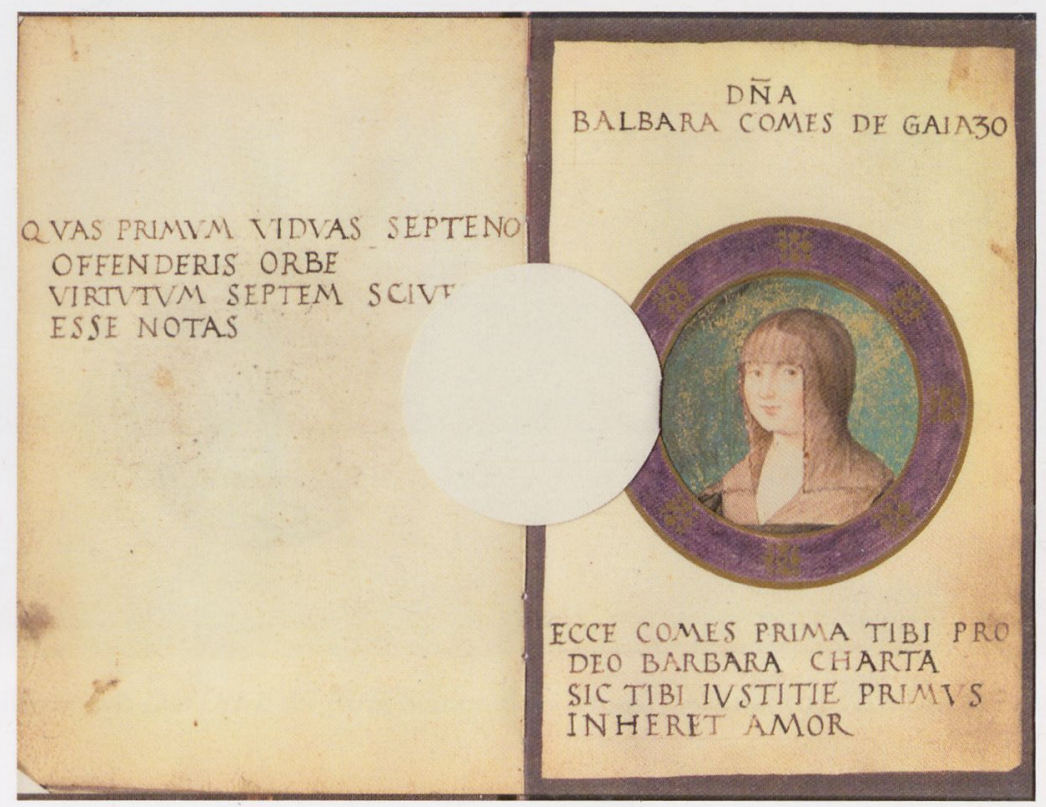

Abbildung 22: Giovanni Ambrogio Noceto: ,Fides: 


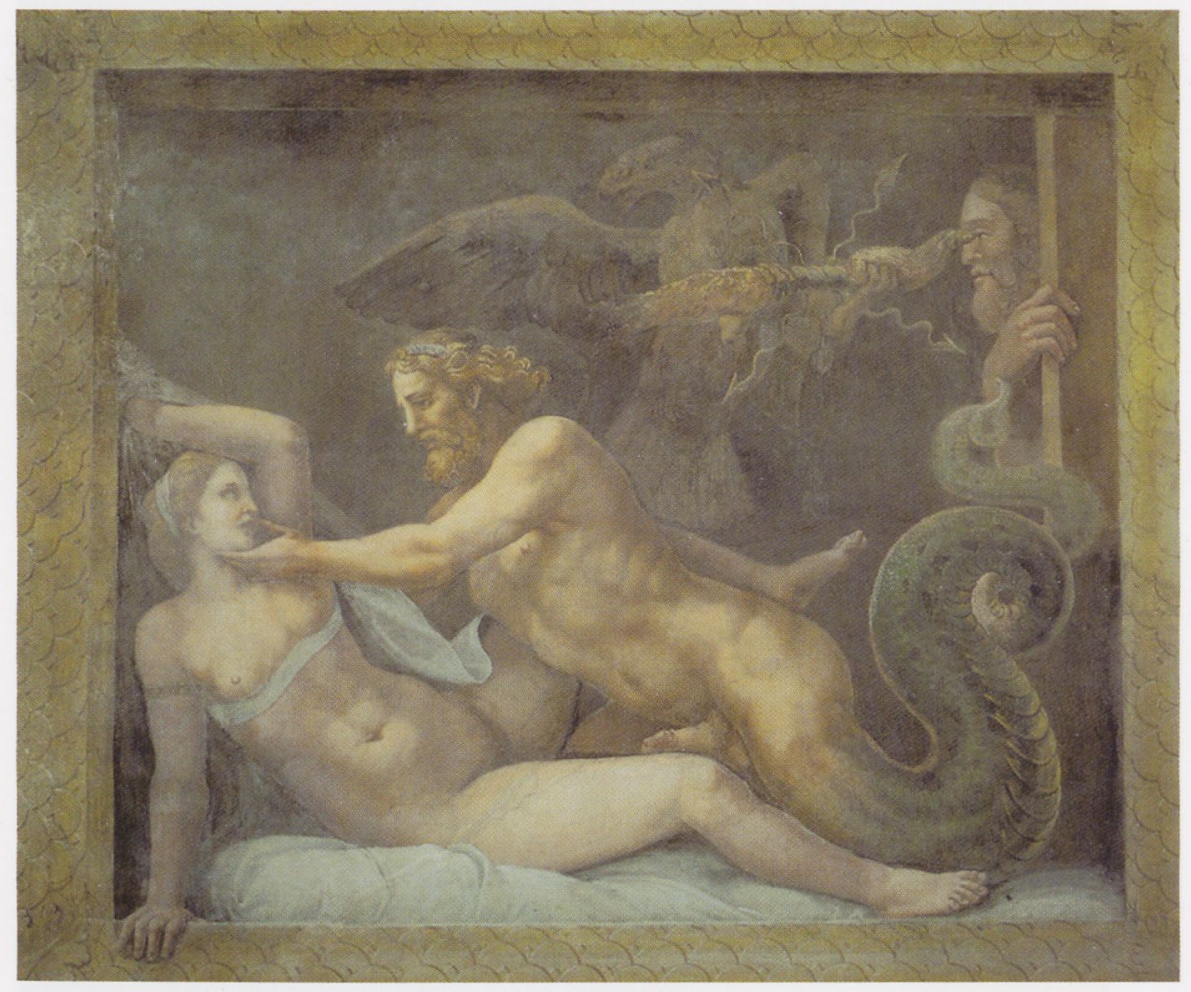

Abbildung 23: Giulio Romano und Werkstatt: Jupiter und Olympias. 


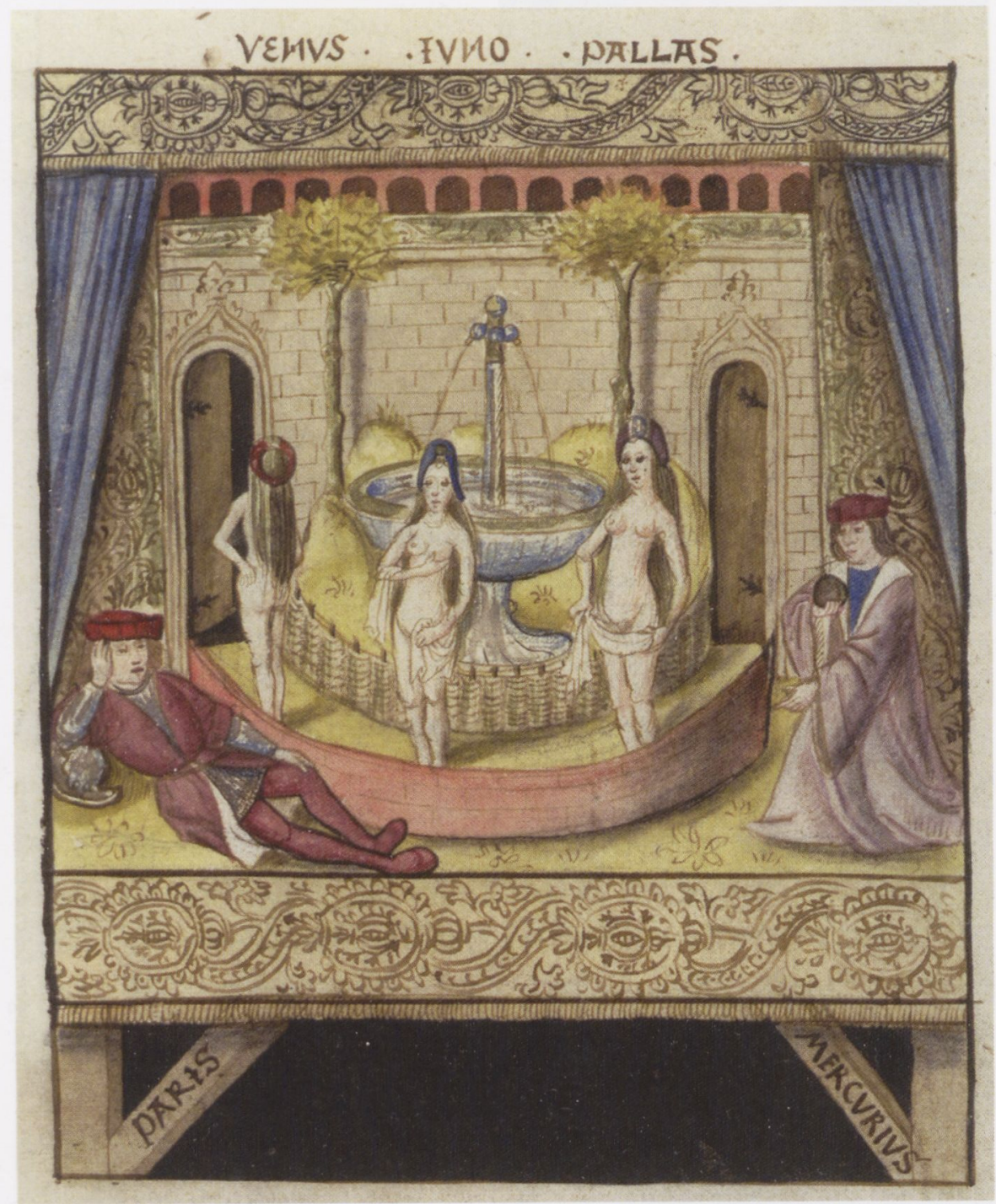

Abbildung 24: Das Urteil des Paris; Station des Einzugs Johannas von Kastilien in Brüssel. 

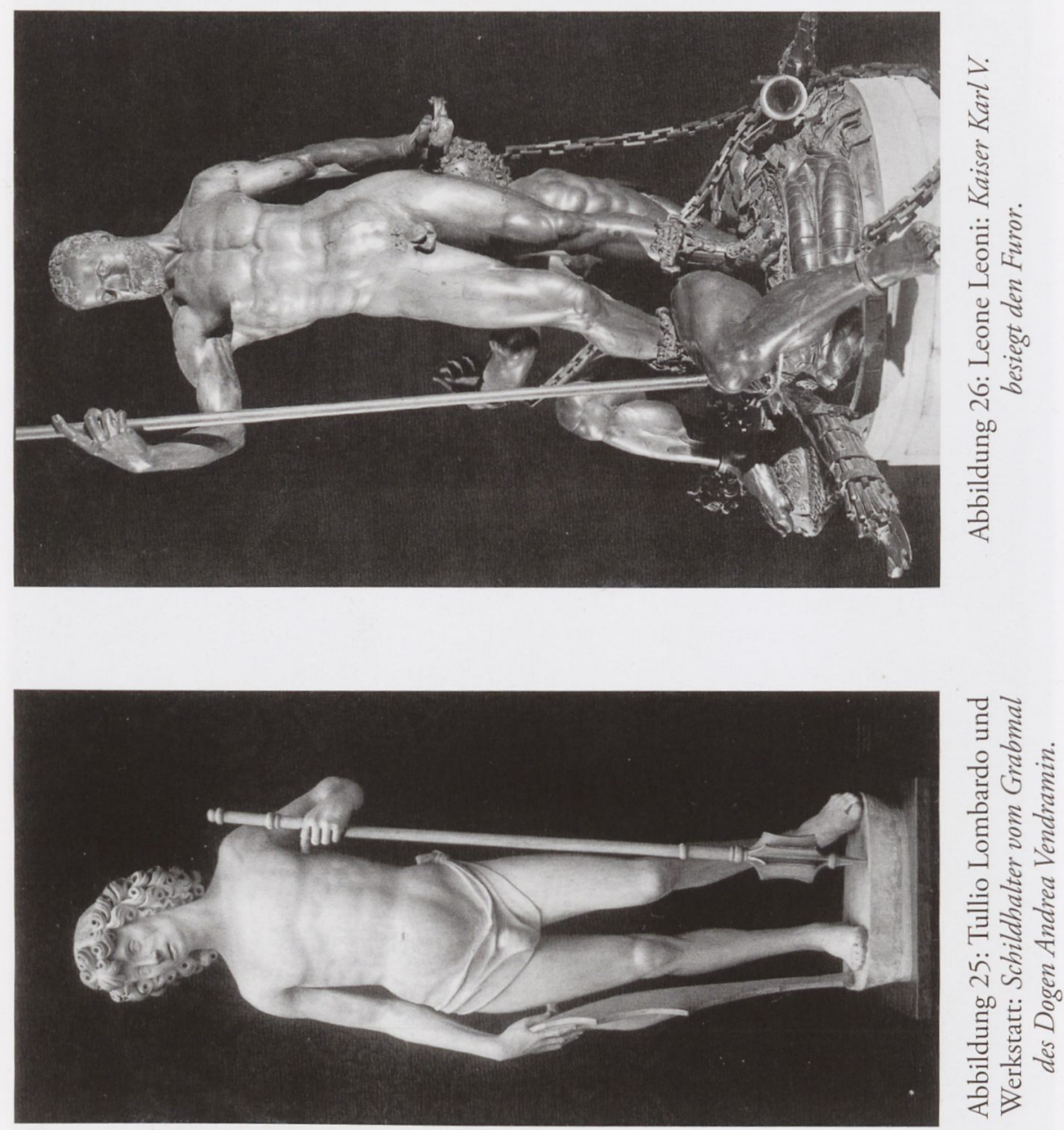
Abbildung 27: Joos van Cleve: Kaiser Maximilian I.

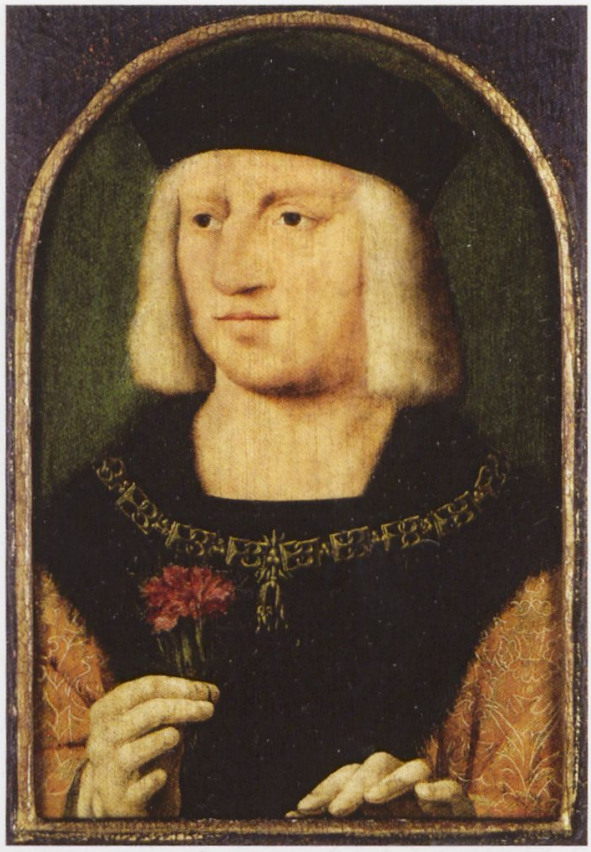

Abbildung 28: Meister von Frankfurt: Kaiser Maximilian I.

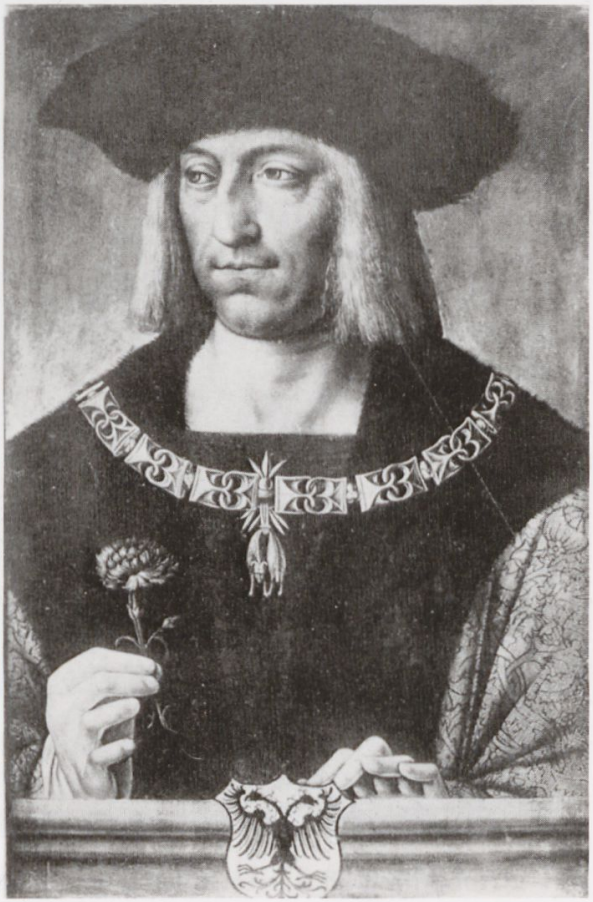




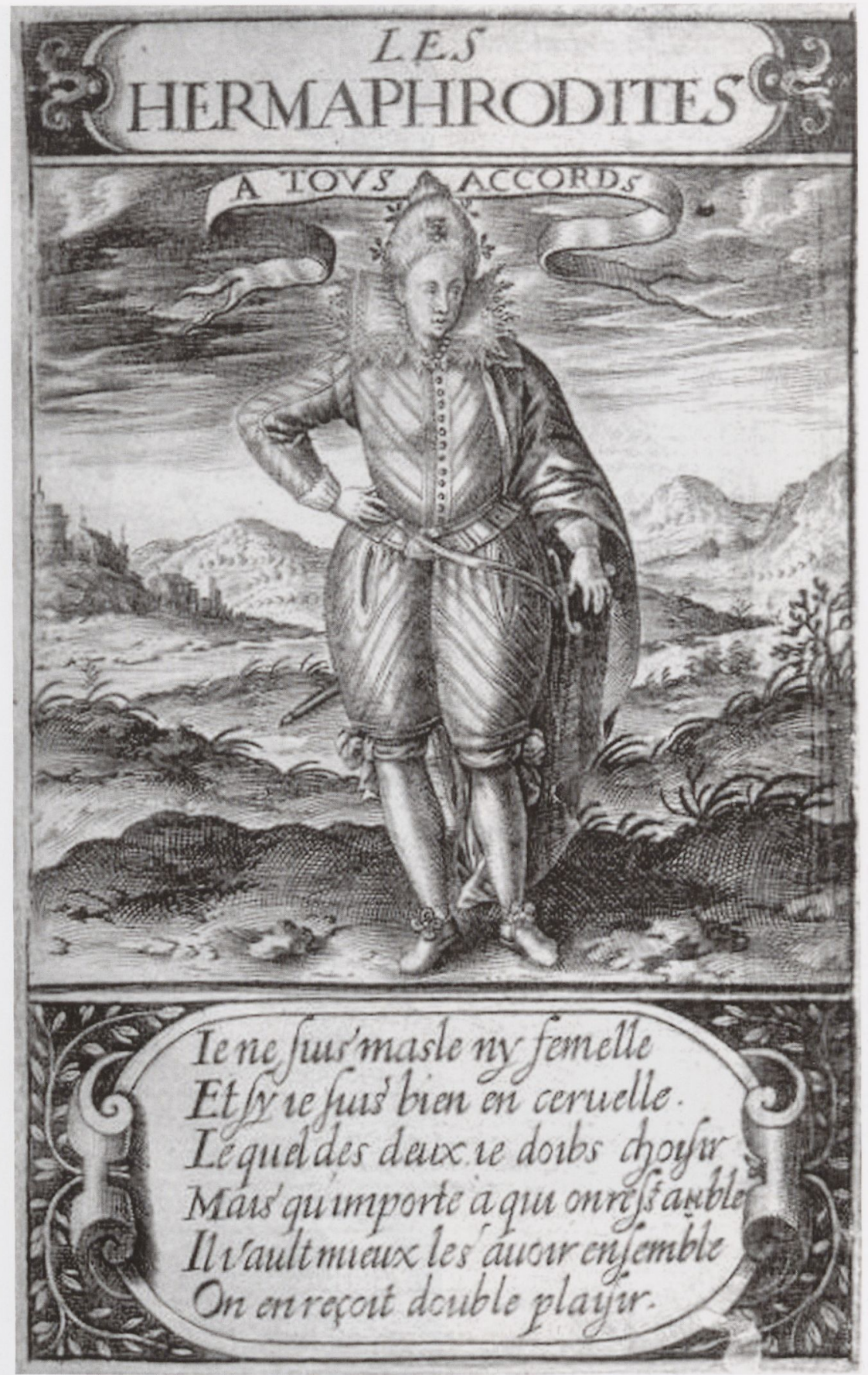

Abbildung 29: Gestochener Titel zu Thomas Artus: L'Isle des Hermaphrodites. 